\title{
Creación de problemas: un método alternativo para introducir y reafirmar el concepto de grupo
}

\author{
Lorena Salazar S. \\ lorena.salazarsolorzano@ucr.ac.cr \\ Escuela de Matemática \\ Universidad de Costa Rica \\ Universidad Nacional de Costa Rica
}

Recibido: 21 Abril, 2014

Aceptado: 12 Julio, 2014

Resumen. En este documento se presenta el diseño de una secuencia de tareas basadas en creación de problemas y uso de material concreto, para introducir el concepto de grupo como estructura algebraica en un curso de álgebra abstracta del plan de estudios de Enseñanza de la Matemática de la Universidad de Costa Rica. Se muestran algunas evidencias que indican que con esto se logra, no solo reafirmar los conocimientos matemáticos, sino también que los futuros docentes desarrollen la habilidad de formular problemas que respondan a un objetivo específico y a su vez reflexionen sobre la actividad matemática.

Palabras clave: Diseño de tareas, creación de problemas, educación matemática, estructuras algebraicas.

\begin{abstract}
This paper describes the design of a task sequence based on creating problems and use of concrete material in order to introduce the concept of group as an algebraic structure, in a course of abstract algebra from the curriculum of Mathematics Education at the University of Costa Rica. Some evidence indicates that this achives, not only to reaffirm the mathematical knowledge, but also that future teachers develop the ability to formulate problems that meet a specific target and reflect on the mathematical activity.
\end{abstract}

KeyWords: Task design, posing problems, mathematics education, algebraic structures. 


\subsection{Introducción}

El origen de esta investigación está relacionado con varios aspectos que me llevaron a reflexionar sobre la necesidad de realizar algunas innovaciones en los cursos tradicionales de matemática formal de la carrera de Enseñanza de la matemática, tanto en la Universidad Nacional de Costa Rica (UNA) como en la Universidad de Costa Rica (UCR), donde imparto cursos de matemática:

- La impartición en la UNA de un módulo sobre resolución de problemas a futuros profesores de matemáticas de secundaria, en el que se discutieron artículos de educadores matemáticos (Ellerton, Malaspina, Van Harpen, Presmeg), los cuales coinciden en que el crear problemas, fomenta el análisis del enunciado y repercute sobre el conocimiento del contenido matemático.

- Las conclusiones de una comisión curricular de la UCR, en la que participé, cuyo objetivo fue el diseño de un plan de estudios para una nueva carrera en Educación Matemática, en la que se hicieron reflexiones sobre el contenido matemático, su abordaje y metodología.

- Las bajas promociones y alta repitencia en los cursos de estructuras algebraicas, a lo largo de mi experiencia docente.

- Errores conceptuales en las pruebas escritas por los estudiantes, que indican carencia de una adecuada asimilación del concepto de grupo como estructura algebraica.

Es por esto que en este trabajo se propone desarrollar el concepto de grupo como estructura algebraica base, usando como y como estrategia el planteamiento de problemas y uso de material concreto, pero no se queda ahí, sino que se propone que el futuro profesor reflexione sobre estos conceptos y su enseñanza. Específicamente, se plantea el siguiente objetivo:

\section{Objetivo}

Diseñar e implementar una secuencia de tareas que permita consolidar el concepto de grupo, como base para las siguientes estructuras algebraicas: anillos y campos, así como sus propiedades fundamentales, mediante uso de material concreto, el planteamiento de problemas, y a su vez, que los futuros docentes, reflexionen sobre la actividad matemática.

La estructura del artículo es la siguiente, después de planter el objetivo de la investigación, a continuación se hace una revisión de la literatura que se ha tenido en cuenta como referentes teóricos. Después se explica la metodología que se ha seguido para pasar, a continuación, a la descripción de la experiencia realizada. El artículo termina con unas consideraciones finales.

\subsection{Nociones teóricas}

A continuación se hacen referencias a algunos elementos teóricos que fundamentaron esta investigación, a saber creación de problemas, constructivismo con uso de material concreto y diseño de tareas. 


\subsubsection{Creación de problemas}

En la última década, las investigaciones en educación matemática centran su atención no solo a la resolución de problemas, sino también al planteamiento de problemas. Por ejemplo Malaspina (2013), afirma que "la creación de problemas está estrechamente ligada a la resolución de problemas y contribuye al desarrollo del pensamiento matemático al brindar oportunidades, a alumnos y profesores, para examinar generalizaciones e iniciarse en la investigación y en el hacer matemáticas". Sin embargo, el "hacer matemáticas", aunque es una competencia importante y necesaria en un futuro profesor de matemática, no es suficiente, también debe tener competencia en el análisis de la actividad matemática, Rubio (2012).

Es importante que los profesores desarrollen competencia en la creación de problemas, al menos en la variación de un problema dado, con el fin de adaptarlo a un objetivo específico. Malaspina (2013), por ejemplo, afirma que el planteamiento de problemas es una estrategia que estimula la capacidad de crear y resolver problemas, lleva a reflexiones didácticas y matemáticas que favorecen el aprendizaje, posibilita encontrar mayores potencialidades que las que se pensaron al crear un problema, muestra la importancia de la redacción de un enunciado y dado que posibilita generalizaciones, lleva a ampliar el horizonte matemático inicial. Sin embargo, hay investigaciones que muestran que los problemas que crean los profesores tienen serias limitaciones, Por ejemplo, Singer y Voica (2013) reportan una investigación con profesores sobre creación de problemas en la que los resultados mostraron porcentajes muy bajos en cuanto a aspectos como claridad, coherencia y originalidad.

En nuestro contexto nacional, que el profesor sea capaz de crear problemas, es fundamental en su futuro profesional, pues los libros de texto usados en secundaria, contienen problemas que usualmente no estan contextualizados a las realidades de los alumnos. Por otro lado, los programas del Ministerio de Educación Pública (MEP 2012), estan fundamentados en la resolución de problemas. Esto hace que en la formación inicial de los profesores de matemática, se incluyan tareas que den al futuro docnete, formación al respecto.

\subsubsection{El constructivismo}

El constructivismo, donde el individuo es responsable de su propio conocimiento, es aplicable a las matemáticas en todos sus niveles. La experiencia de esta investigación se centra en el ámbito universitario, con un doble propósito: que el estudiante aprenda el concepto matemático involucrado, pero que además la actividad le resulte un foco de reflexión para su futura actividad profesional, como docente de secundaria. Según Kilpatrick, Gómez y Rico (1995), mencionado por Castillo (2008), refiriéndose a como se construye el conocimiento matemático, señalan que:

- El conocimiento matemático es construido, al menos en parte, a través de un proceso de abstracción reflexiva.

- Existen estructuras cognitivas que se activan en los procesos de construcción.

- Las estructuras cognitivas están en desarrollo continuo.

- La actividad con propósito induce la transformación de las estructuras existentes. 
Según Castillo (2008), "el individuo que aprende matemáticas desde un punto de vista constructivista debe construir los conceptos a través de la interacción que tiene con los objetos y con otros sujetos. Tal parece que para que el alumno pueda construir su conocimiento y llevar a cabo la interacción activa con los objetos matemáticos es preciso que dichos objetos se presenten inmersos en un problema, no en un ejercicio. "

Con base en estos investigadores, la actividad reportada en este documento, presenta la aplicación de diferentes tareas diseñadas con el objetivo de que el estudiante, asimile el concepto de grupo: creando grupos, primero en los conjuntos numéricos, y luego en conjuntos no tan familiares a ellos, mediante manipulación de material concreto.

\subsubsection{Diseño y secuencia de tareas}

Muchos investigadores en educación matemática (Sullivan, Clarke y Clarke, 2013), han manifestado interés en el diseño de tareas que respondan a un objetivo específico. En el 2013 se desarrolló un ICMI Study específico sobre este tema (Margolinas, 2013), con el fin de contestar preguntas relacionadas a como diseñar una secuencia de tareas pertinentes.

En una clase convencional de estructuras algebraicas, usualmente el docente se limita a exponer, mediante clases magistrales y a un ritmo acelerado, la definición de grupo, seguido inmediatamente de ejemplos, teoremas y resultados sobre sus principales propiedades, para proseguir con ejercicios propuestos. Después de seguir esta metodología en repetidas ocasiones, se fue haciendo más evidente que en realidad el concepto de grupo, que es la base del resto de las estructuras algebraicas, en muchos casos, no estaba claro en los estudiantes. Lograr la comprensión y la articulación de las diferentes estructuras algebraicas, requieren un mayor esfuerzo por parte de los estudiantes, pero también se requiere un mayor esfuerzo por parte del docente, pues debe romper con el esquema de enseñanza tradicional, donde usualmente él es un transmisor de conocimientos y el estudiante es un receptor pasivo, que se supone debe asimilar los conceptos de esta manera. Debe pasar de ser un simple emisor, a ser un creador y organizador de una secuencia de tareas con un fin específico.

Lamentablemente, no es habitual que los docentes del área de matemática formal, diseñen secuencias de tareas con el objetivo de facilitar la comprensión de los objetos matemáticos en general y la articulación coherente de los componentes involucrados. Tampoco es habitual diseñar tareas que desarrollen competencias de reflexión sobre la matemática en los futuros docentes de secundaria. Por ejemplo, Rico (2004) expone diez competencias específicas, consensuadas por formadores de profesores e investigadores en educación matemática, que participaron de un seminario celebrado en Granada, España, dedicado al análisis y diseño de las competencias que debe tener un profesor de matemática de secundaria. Entre ellas destacan algunas relacionadas a esta investigación:

- Reconocer los tipos de razonamiento de los estudiantes.

- Proponer tareas que los orienten.

- Diagnosticar sus errores, y proponer los correspondientes procesos de intervención.

- Seleccionar y secuenciar actividades para el aprendizaje escolar. 
- Analizar los diversos problemas que surgen en situaciones de aprendizaje.

- Utilizar técnicas de comunicación para dotar de significado los conceptos matemáticos.

- Conocer recursos y materiales (computacionales, audiovisuales, manuales, bibliográficos, etc.) y emplearlos adecuadamente en la enseñanza de las matemáticas de secundaria.

Otros investigadores, Giménez, Font \& Vanegas (2013), reflexionan sobre las tareas que permiten el desarrollo de la competencia de análisis didáctico en la formación de futuros profesores de matemáticas de secundaria. En este trabajo se pretende reflexionar sobre dos de los seis tipos de tareas propuestos por ellos: "análisis de prácticas, objetos y procesos matemáticos y propuesta de mejora justificada de esas prácticas", refiriéndose en este caso a las "prácticas", como a las consignas o tareas propuestas en las actividades diseñadas. Es decir, se pretende que los alumnos hagan el papel dual: de estudiantes aprendices y de analistas de una práctica pedagógica. Aspecto importante que debe ser considerado en la formación inicial de estos profesionales.

\subsection{Metodología}

La aplicación de las tareas diseñadas, tuvieron lugar a inicios del I ciclo del 2014, donde el primer tema a desarrollar es el concepto de grupo, en el curso Álgebra para la enseñanza de la carrera de Enseñanza de la Matemática de la Universidad de Costa Rica, la cual forma profesores de matemática para educación media. Este curso está ubicado en el I ciclo del IV año y tiene una modalidad presencial con 5 horas de clases por semana. El mismo introduce los conceptos básicos de estructuras algebraicas: grupos, anillos y campos, sin perder de vista su tratamiento formal, dando pruebas y definiciones de manera rigurosa.

Participaron 21 estudiantes del curso mencionado anteriormente (19 estudiantes regulares y dos estudiantes de sedes regionales), que en su gran mayoría estan llevando el curso por primera vez, lo cual lo hace ideal para la experiencia y para concluir sobre si las actividades realizadas, tienen un impacto positivo o no. Cada estudiante ingresa a un curso con una nota llamada promedio ponderado, que es el promedio de las notas finales obtenidas en cursos anteriores, aprobados o no. La gráfica 1.1 nos da una idea del promedio ponderado de los integrantes regulares del grupo, (no se tomó en cuenta los dos estudiantes de sedes regionales), donde se realizó la actividad. Se puede ver que la mayoría se encuentra entre un promedio entre 6.0 y 8.0. Cabe aclarar que la nota en la Universidad de Costa Rica se encuentra entre 0 y 10, siendo 7.0 lo mínimo para aprobar un curso. 


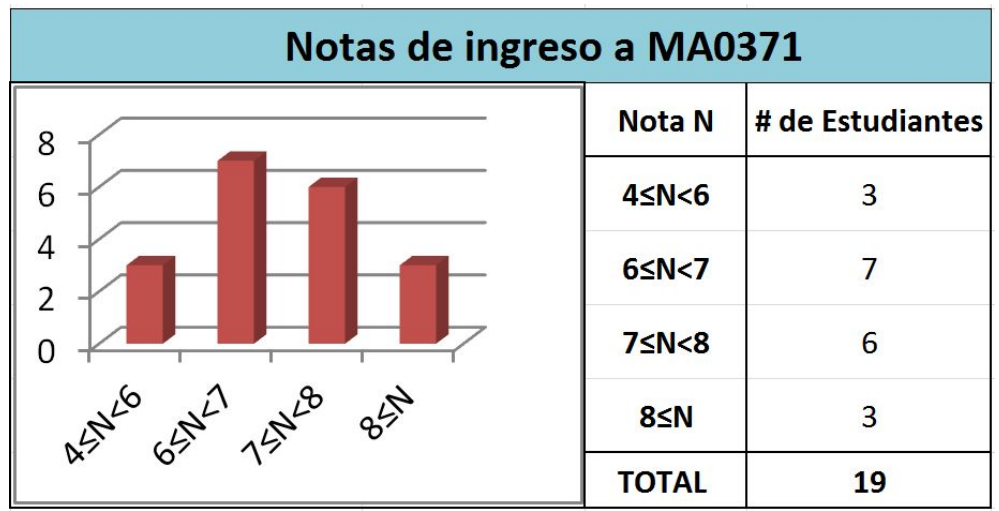

Figura 1.1: Promedio ponderado de los estudiantes del curso

\subsubsection{Técnicas e instrumentos de recolección de datos}

Para la recolección de datos, se usó la observación no participante y registro detallado, para lo cual se usó un diario donde se fue anotando todo lo que fue ocurriendo en el aula: percepciones sobre la actitud e interés de los estudiantes, expresiones verbales de los participantes, tiempo de ejecución de las tareas, etc. Se recolectaron además, evidencias escritas por los grupos de trabajo en el desarrollo de la actividad realizada. Entre las tareas asignadas, se les solicitó evaluar la actividad, desde la óptica del futuro docente que será cada uno de ellos. Para que tuvieran un argumento sólido y capacidad de debate, se les asignó realizar una lectura sobre creación y planteamiento de problemas, más una actividad donde pudieran plasmar diseño de tareas en un tema específico de secundaria. Finalmente se les aplicó una prueba escrita, para evaluar el concepto de grupo. Todos estos elementos, ayudaron a evaluar la actividad.

\subsubsection{Diseño de tareas para la actividad}

Para la implementación de la experiencia, se diseñaron tres bloques, cada uno de ellos agrupa varias consignas o actividades en los que se especifica: intención de las consignas, contenidos matemáticos, instrucciones para el estudiante y lo que se espera que el estudiante realice.

\section{Bloque 1}

Propósito: Este bloque pretende que los estudiantes asimilen el concepto de grupo como estructura algebraica base, mediante reflexión detallada sobre cada una de las partes involucradas en su definición. Se pide asímismo, la creación de grupos en conjuntos familiares a ellos.

Contenidos: Conceptos de operación binaria, grupo, neutro e inversos.

Resultado esperado: Se espera que el estudiante refuerce, mediante creación de grupos, los conceptos de cerradura, asociatividad, elemento neutro, unicidad, los inversos, etc.

Tiempo: 1.5 horas.

Consignas: 0 a 2 
Bloque 2

Propósito: Este bloque pretende que los estudiantes implementen tablas de grupos de simetrías de polígonos regulares, mediante manipulación de material concreto, para simular rotaciones y simetrías de las figuras.

Contenidos: Concepto de grupo, concepto del neutro, de los inversos, movimientos rígidos del plano.

Resultado esperado: Este bloque pretende propiciar que el estudiante descubra, con base en su propia manipulación del material, los grupos de simetrías algunos polígonos regulares y logren una generalización intuitivamente, a cualquier orden.

Tiempo: 2.5 horas.

Consignas: 3 a 6 .

\section{Bloque 3}

Propósito: Este bloque pretende que los estudiantes asimilen, con más convicción, la prueba formal, de lo que la intuición y la construcción de tablas de grupos, les dejó respecto a los grupos de simetrías de cualquier polígono regular de orden $n$.

Contenidos: Grupos de simetrías, movimentos rígidos del plano, conceptos de neutro, inversos, composición de movimientos.

Resultado esperado: Este bloque pretende que los estudiantes comprendan la prueba formal de los grupos de simetrías.

Tiempo: 2.5 horas.

Consignas: 7 a 9.

\section{Bloque 4}

Propósito: En este bloque se pretende que el estudiante haga una valoración y un análisis reflexivo de la actividad.

Contenidos: Análisis didáctico, reflexión de la práctica, diseño de tareas.

Resultado esperado: Se espera que los estudiantes diseñen consignas para responder a un objetivo específico sobre algún tema de matemática de los programas de secundaria.

Tiempo: 4 horas (extra clase) 
Consignas: 10 a 12.

\subsection{Descripción de la experiencia}

A continuación se detallan tanto las consignas o tareas diseñadas para el logro de los objetivos, como algunos comentarios resultado de la aplicación o implementación en el aula. Antes de cada consigna se hace un comentario sobre la intención de la misma y lo que se espera de ella.

\subsubsection{Consignas del bloque 1:}

Dado que en experiencias anteriores de enseñanza, se ha notado que aún en este nivel, tienen problemas de conexión y aplicación de la lógica formal, se inició con una consigna para que reflexionaran entre las diferencias de orden de los cuantificadores al analizar la veracidad de validez de una proposición $P(x, y)$. Esta es la idea de la primera consigna, a la que se le ha numerado con 0 , porque podría considerarse opcional, dependiendo si se considera necesario o no.

\section{Consigna 0:}

Discuta con sus compañeros, ¿cuál es la interpretación de $\forall x \forall y P(x, y)$ ? ¿Cuándo es verdadera esta expresión? ¿Cuándo es falsa? Haga lo mismo con $\forall x \exists y P(x, y), \exists x \forall y P(x, y), \exists x \exists y P(x, y)$. Construya un ejemplo para mostrar que en general $\forall x \exists y P(x, y)$ y $\exists x \forall y P(x, y)$, tienen significados diferentes.

Se introdujo la consigna anterior, dado que algunos estudiantes, en cursos pasados, confunden hechos como que el neutro del grupo es un elemento único, y debe "neutralizar" a todos los elementos del conjunto, mientras que el inverso es único para cada elemento del conjunto.

La siguiente consigna, intenta, que en lugar de que el docente escriba y explique la definición de grupo, se les de una ficha con esta, de modo que discutan y asimilen por sí mismos, este concepto antes de proceder a niveles superiores de dificultad.

\section{Consigna 1:}

Exprese con sus propias palabras qué entiende por cada uno de los puntos 1, 2 y 3 en la definición de grupo siguiente. Ponga atención a los cuantificadores $\exists$ y $\forall$ involucrados en la misma. Definición de Grupo

Un grupo $(G, *)$ es un conjunto cualquiera $G$ con una operación $(*)$, usualmente llamada operación suma, aunque no se relaciona con alguna suma que usted conozca, tal que:

1) La operación $*$ es cerrada y asociativa

2) Existe un elemento $e \in G$ tal que $g * e=g=e * g, \quad \forall g \in G$.

3) Para cada elemento $g \in G$, existe un elemento $g^{\prime} \in G$ tal que $g * g^{\prime}=e=g^{\prime} * g$

Dada que esta es la primera consigna, y probablemente la primera vez que los estudiantes reflexionarán sobre una definición, se diseñó una guía de preguntas con la intención de que los estudiantes tomen 
conciencia, cada vez que lean una definición, enunciado de algún teorema o resultado, de prestar atención a los detalles y cada una de las partes involucradas en la misma.

\section{Guía de preguntas para consigna 1:}

- ¿Qué significa ese par $(G, *)$ ? ¿Es la notación que se va a usar para denotar un grupo?

- El conjunto $G$ debe ser ¿de números? ¿de letras? ¿de funciones? ¿de figuras geométricas? ¿podría ser de los integrantes de éste grupo de trabajo?

- ¿qué es una operación? ¿que significa operación binaria? ¿Es cerrada?

- ¿qué significa la propiedad de asociatividad? Entonces la operación debe ser cerrada y asociativa, ¿porqué cerrada?

- ¿Qué significa que se satisface $g * e=g=e * g, \quad \forall g \in G$. ? ¿Porqué cree usted que el elemento " $\mathrm{e}$ " recibe el nombre de neutro? ¿satisfice éste elemento, la propiedad mencionada con todos los elementos de G? ó ¿para cada elemento de G, hay un neutro?

- ¿Qué significa que se satisface $g * g^{\prime}=e=g^{\prime} * g$. ? ¿Porqué cree usted que el elemento recibe el nombre de inverso de $\mathrm{g}$ ? ¿satisfice éste elemento la propiedad mencionada con todos los elementos de G? ó ¿para cada elemento de $G$, hay un inverso?

- Finalmente conteste la pregunta de la consigna 1, qué es entonces un grupo, en sus propias palabras.

Esta consigna llevó mucho más del tiempo estipulado para ello. Sin embargo, les ayudó a la comprensión de cada uno de los puntos de la definición de grupo. Algunos hasta este punto, comprendieron que cada elemento de un grupo debe tener su propio inverso.

Una vez asimilada la definición de grupo, la siguiente consigna pide construir grupos, primero en conjuntos finitos pequeños, y luego en conjuntos numéricos conocidos. También hay inmersa la idea de que vayan introduciendose en el concepto de isomorfismos de grupos. Esta es la primera tarea en la que se le pide que cree un grupo, bajo la modalidad de creación de problemas.

\section{Consigna 2:}

- Defina, de ser posible, una operación en cada uno de los conjuntos siguientes de modo que se forme un grupo:
a) $\{$ bola $\}$,
b) $\{0,1\}$,
c) $\{1,-1\}$,
d) $\{a, b\}$,
e) $\{0,1,-1\}$,
f) $\{a, b, c\}$.

- Haga lo mismo con los conjuntos infinitos $\mathbb{N}, \mathbb{Z}, \mathbb{Q}, \mathbb{R}$.

Con el conjunto $G=\{$ bola $\}$, tuvieron problemas para definir una operación. Algunos dejaron de lado la cerradura, definiendo, erróneamente,

$$
\text { bola }+ \text { bola }=2 \text { bola }
$$




\section{$0+6=0$}

Figura 1.2: Operación en un grupo unitario

Al indicarles que el elemento 2 bola no pertenecía al conjunto, hubo un poco de confusión, pues contestaron que tenía que estar en el conjunto, precisamente por la cerradura. Esto se aprovechó para hacer una reflexión a nivel de todo el grupo sobre la definición, nuevamente. Efectivamente bola + bola debe estar en el conjunto, y como el único elemento de $G$ es bola, pues no queda otra alternativa más que definir

$$
\text { bola }+ \text { bola }=\text { bola }
$$

Así, tenemos la primera sorpresa! Las cosas en los grupos no resultan como se espera que ocurrieran.

Se reflexionó sobre la importancia de apegarse muy bien a la definición y sobre lo que es en general una definición en matemática, y no asumir cosas que no se digan explícitamente. También se reflexionó sobre la operación + , en un grupo la operación es una idea abstracta que une dos elementos y da un tercero, pero que la simbología no debe apegarse a una idea preconcebida, en este caso la operación + es solo un símbolo, no se refiere a la suma usual de conteo en los números naturales.

Se les indicó que de ahora en adelante, se usaría este símbolo, en la mayoría de los casos, para referirse a la operación del grupo.

Una vez aclarado esto, se les pidió que ahora pasaran de ser alumnos a ser profesores, es decir que se visualizaran como lo que pronto les tocaría enfrentar en su labor profesional. Se les hizo ver, que no esperaba que la discusión hubiera llegado al punto que llegó, que esto no era algo que se planeó con antelación. Se les solicitó que analizaran la discusión dada, las respuestas de la docente, como había conducido la discusión y sobre como la mejorarían ellos. Esto dio pie a más reflexiones y comentarios importantes desde el punto de vista formativo, como por ejemplo el de uno de ellos que expresó: "profesora ahora veo que uno tiene que estar alerta, pues no se sabe que estará pasando por la cabeza del muchacho, uno cree que están entendiendo, y tal vez no tienen ni idea”. Otra muchacha dijo: “ uno puede planear una clase muy linda, y no sabe que puede pasar luego en el aula", lo cual es perfectamente una realidad que deberán enfrentar los profesores. 


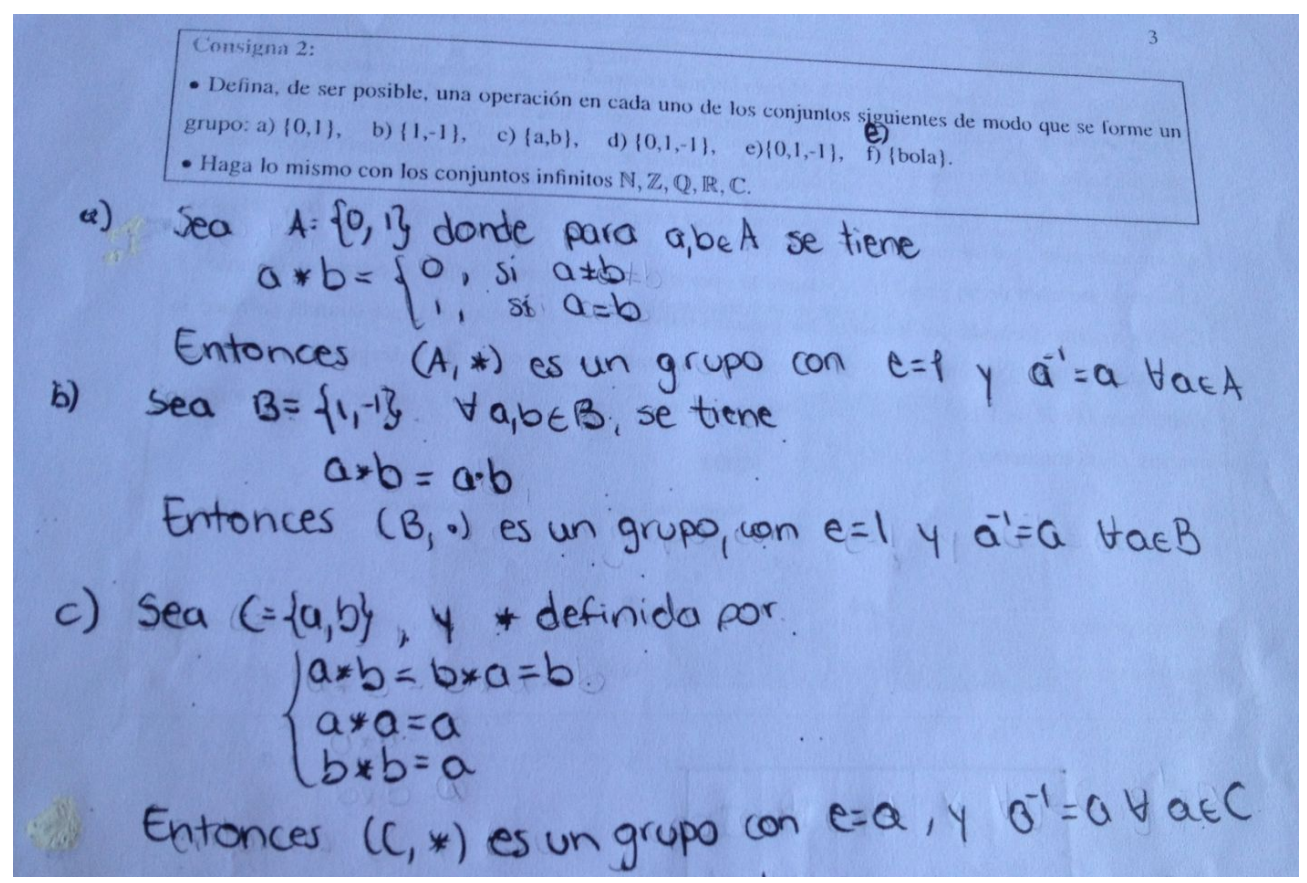

Figura 1.3: Creación de grupos de orden dos

Al continuar con los siguientes conjuntos de la consigna anterior, uno de los grupos definió una operación para cada uno de los conjuntos $\{0,1\},\{1,-1\}$ y $\{a, b\}$, como se muestra en la figura 1.3. Observe que en el caso del conjunto $\{0,1\}$, después de intentar con la suma usual de números enteros, la cual no resulta cerrada cuando se opera $1+1$, definen otra operación como 0 si son iguales y 1 si son diferentes. Para el conjunto $\{1,-1\}$ definen la operación producto de números enteros, y para $\{a, b\}$, definen la operación como $a$ si son iguales y $b$ si son diferentes, repitiendo el argumento usado en el conjunto $\{0,1\}$.

Al preguntarles si los tres eran grupos diferentes, ellos contestaron, un poco extrañados por la pregunta, que claro que eran diferentes dado que la operación era diferente y los elementos eran diferentes. Se les hizo plantear los grupos creados usando una tabla, como se muestra en la figura 1.4, para que se les facilitara notar la similitud de las mismas, y que si miraban de una forma diferente, es decir viendo solo la estructura de los grupos, en realidad los tres eran el "mismo" grupo.

Esto se hizo con el fin de ir introduciendo el concepto de isomorfismo. También se les hizo la observación de que este hecho, sí se había planeado y anticipado, razón por la cual se había incluido en la consigna anterior. Esto con el fin, de ir logrando la reflexión sobre la actividad matemática, e ir mostrando un modelo de enseñanza, donde todo debe estar justificado y preparado de antemano. Aunque es claro que siempre pueden ocurrir, y pasan constantemente, hechos no previstos por el docente. 


\begin{tabular}{|c|c|c|}
\hline$*$ & 0 & 1 \\
\hline 0 & 1 & 0 \\
\hline 1 & 0 & 1 \\
\hline
\end{tabular}

\begin{tabular}{|r|r|r|}
\hline & 1 & -1 \\
\hline 1 & 1 & -1 \\
\hline-1 & -1 & 1 \\
\hline
\end{tabular}

\begin{tabular}{|c|c|c|}
\hline$\otimes$ & $a$ & $b$ \\
\hline$a$ & $a$ & $b$ \\
\hline$b$ & $b$ & $a$ \\
\hline
\end{tabular}

Figura 1.4: Tablas de grupos isomorfos de orden dos

LLama la atención el hecho de que un grupo de estudiantes intentó definir una operación en $\mathbb{N} \cup\{0\}$, que no resulta ser un grupo, como puede verse en figura 1.5. Es importante aprovechar algunos errores, para recalcar aspectos que reafirmen algunos conceptos. De los errores se puede lograr un aprendizaje significativo.

\section{Grupos para los conjuntos $\mathbb{N}, \mathbb{Z}, \mathbb{Q}, \mathbb{R} y \mathbb{C}$ \\ a) $G=\{\mathbb{N}\}$, donde $(G, *)$

$$
\forall a, b \in \mathbb{N} \text { Se tomara } a * b= \begin{cases}a & \text { si } b<a \\ 0 & \text { si } b=a \\ b & \text { si } a<b\end{cases}
$$

\section{Se obtiene}

- Cerradura.

- Neutro: 0

- Inverso: El inverso de un número es sí mismo.

Figura 1.5: Una operación que no hace de $\mathbb{N} \cup\{0\}$ un grupo

\subsubsection{Consignas del bloque 2:}

La siguiente consigna pide que construyan grupos en conjuntos no comunes a ellos, pero usando material concreto y manipulable. La idea es que los estudiantes logren crear las tablas de los grupos de simetrías de polígonos regulares.

Para ello se les proporcionó a cada estudiante "alfombras rompecabezas", como se muestra en la figura adjunta, numerados del 1 al 9. No se les indicó como usar el material dado.

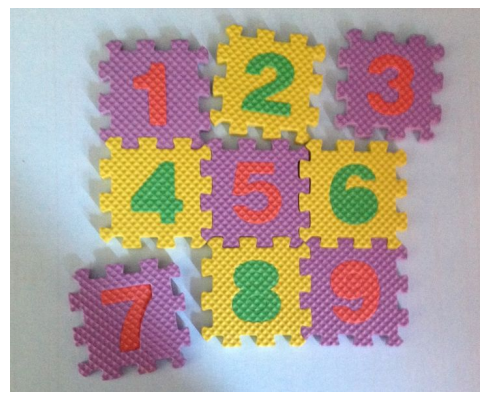

Figura 1.6: Material concreto

A otros grupos se les proporcionó, el mismo material pero con letras seguidas del abecedario, en lugar de números, con el fin de que solo vieran estructura y características propias de los elementos. 


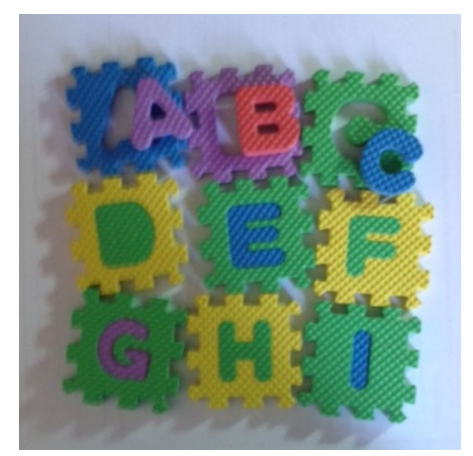

Figura 1.7: Material

Algunos tuvieron un poco de dificultad, pues las instrucciones se referían a los números 1,2 3 y 4, pero se les indicó que este acomodo era parte del ejercicio, pues la idea es ir introduciéndoles el concepto de isomorfismos de grupos, de modo que la apariencia de los elementos no importa.

\section{Consigna 3: Un juego!}

Se tienen un cuadrado con vértices numerados con $\{1,2,3,4\}$.

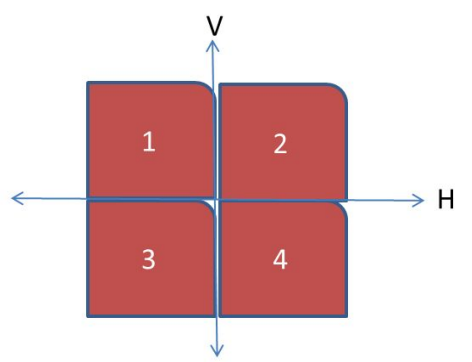

Construya un un grupo $(G, \circ)$ donde la operación o es la composición de movimientos simétricos con respecto a rectas que mantengan la figura del cuadrado invariante, y $G$ es el conjunto formado por los todos los posibles movimientos. Haga una tabla del grupo creado e indique cuál es el neutro y los inversos de cada movimiento.

Al principio, los estudiantes muy entusiasmados con el material concreto, no sabían como empezar, poco a poco fueron creando ideas de como usar el material concreto dado, para generar los movimientos solicitados. Una dificultad que se presentó en todos los grupos, fue el poder plasmar por escrito, los movimientos y su combinación. ¿Cómo representar una simetría vertical? En este momento, la docente intervino sugieriéndoles darle nombres sugestivos a cada movimiento, por lo que les solicitó elegir una notación adecuada. Por consenso, el grupo decidió usar la siguiente notación:

Simetría vertical: con $(\mathrm{V})$, por vertical

Simetría horizontal: con $(\mathrm{H})$, por horizontal

Movimiento diagonal: con (D), por diagonal 
Movimiento nulo: con (I), para indicar que la figura se deja igual.
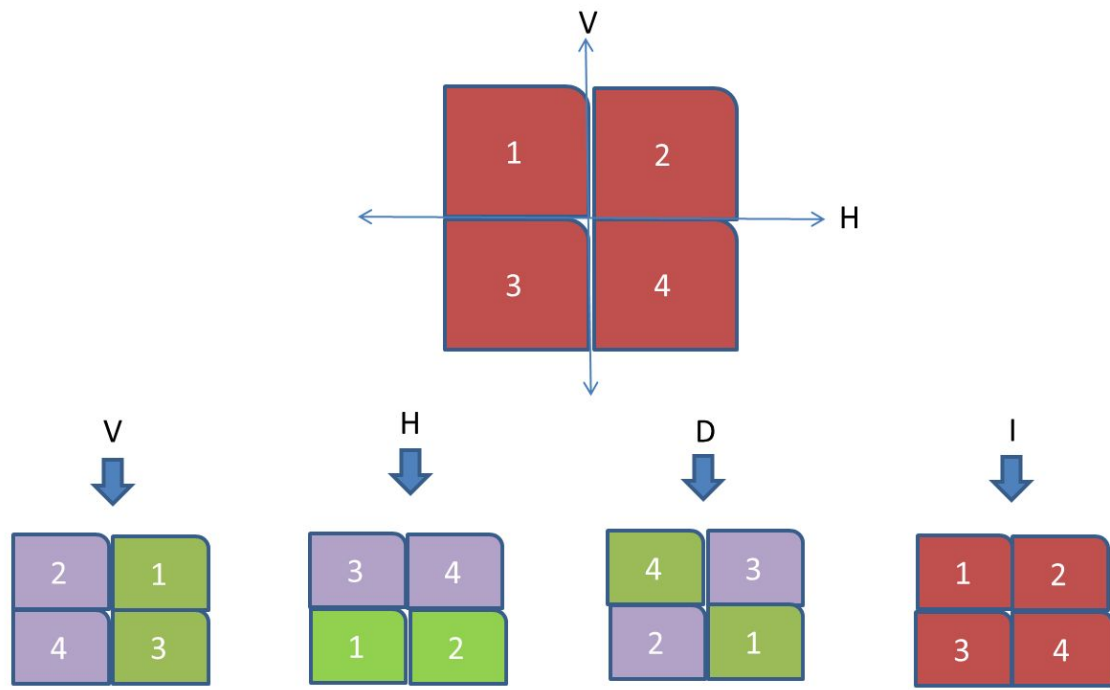

Figura 1.8: Movimientos respecto a simetrías del cuadrado

Tuvieron un poco de problemas al hacer las primeras combinaciones, pero poco a poco todos lograron el objetivo. Algo que llama la atención es que cada vez que iniciaban una combinación de movimientos, ponían la figura en su posición inicial. Aunque la docente les indicaba que podían realizar el movimiento desde cualquier posición en que se hallaban la numeración de las alfombritas, parecía que no comprendían, hasta verificarlo ellos mismos y anotar en la tabla.

De modo que la combinación de los movimientos (simetría diagonal) ○ (simetría horizontal), se representará como $D \circ H$, y eso es lo mismo que $V$, como puede verse en la figura 1.9.

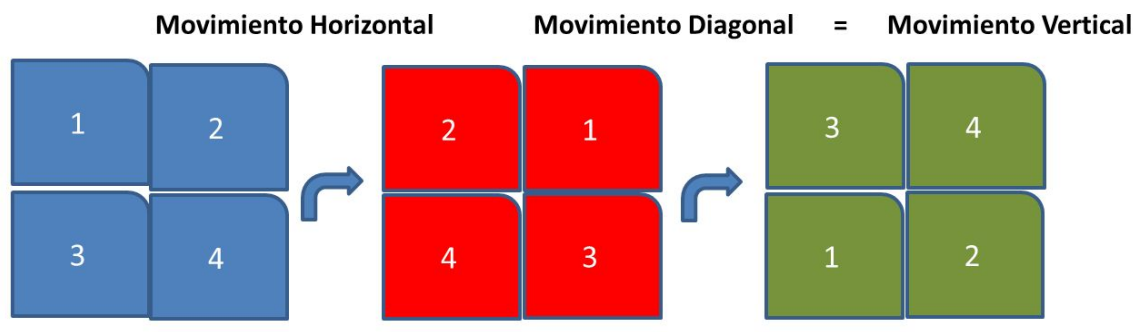

Figura 1.9: Movimiento $D \circ H=V$

Una vez superada la dificultad de notación, los estudiantes avanzaron muy rápido formando todas la posibles combinaciones de movimientos ir plasmando en una tabla los resultados. Determinaron el movimiento que funciona como "identidad", así como los movimientos inversos de cada uno de ellos. Hicieron algunos casos de la asociatividad. A continuación puede apreciarse la tabla 1.10 creada por uno de los grupos, que refleja los movimientos con respecto a las simetrías indicadas. 


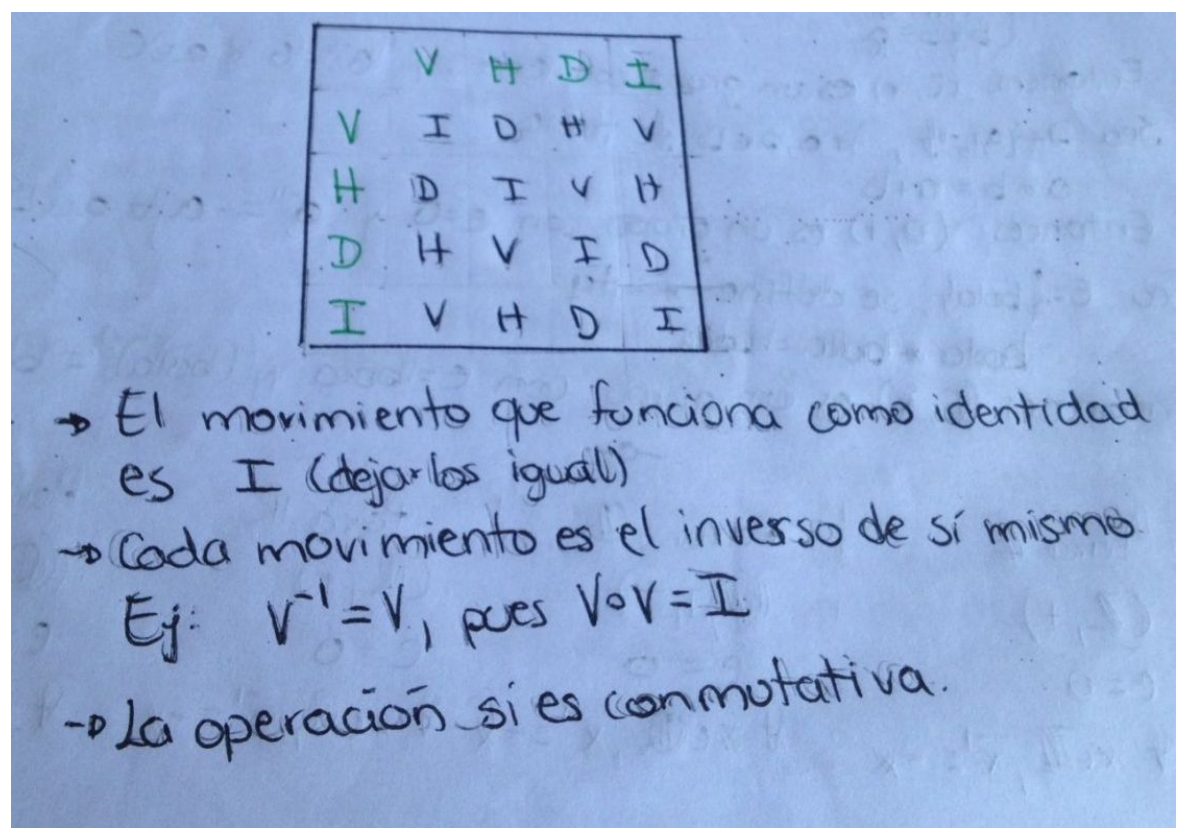

Figura 1.10: Ejemplo de un grupo creado

En esta siguiente consigna, se les solicitó hacer una variación al problema anterior, con un grado de dificultad mayor, hay más movimientos incluidos.

\section{Consigna 4: Una variación al juego anterior}

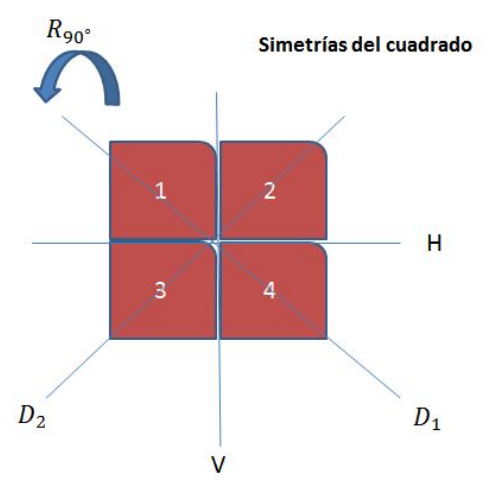

Ahora incluya, además de los movimientos vertical y horizontal del problema anterior, rotaciones de $90^{\circ}$ en sentido contrario a las manecillas del reloj, y en lugar del movimiento vertical de antes, sepárela en dos simetrías con respecto a las diagonales del cuadrado.

Construya una tabla de un grupo $(G, 0)$ donde la operación o es la composición de estos movimientos y $G$ es el conjunto formado por los todos los posibles movimientos. ¿Hay ahora movimientos repetidos? Identifique cuál movimiento funciona como identidad y cuáles son movimientos inversos. ¿Será conmutativa la operación? 
Una vez pasada la consigna dos, esta ya no generó mayor problema al incluir ahora como nuevos movimientos las rotaciones y dos simetrías diagonales, en lugar de solo una. Uno de los grupos usó la siguiente notación:

$D_{1}$ : que consiste en dejar 1 y 4 fijos e intercambiar 2 y 3 ,

$D_{2}$ : que consiste en dejar 2 y 3 fijos e intercambiar 1 y 4 .

$R_{1}$ : que corresponde a una rotación de $90^{\circ}$.

$R_{2}$ : que corresponde a una rotación de $180^{\circ}$.

$R_{3}$ : que corresponde a una rotación de $270^{\circ}$.

$R_{4}$ : que corresponde a una rotación de $360^{\circ}$.

$H$ : que consiste en cambiar 1 con 3 y 2 con 4.

$V$ : que consiste en cambiar 1 con 2 y 3 con 4.

I: que consiste en dejar todo igual
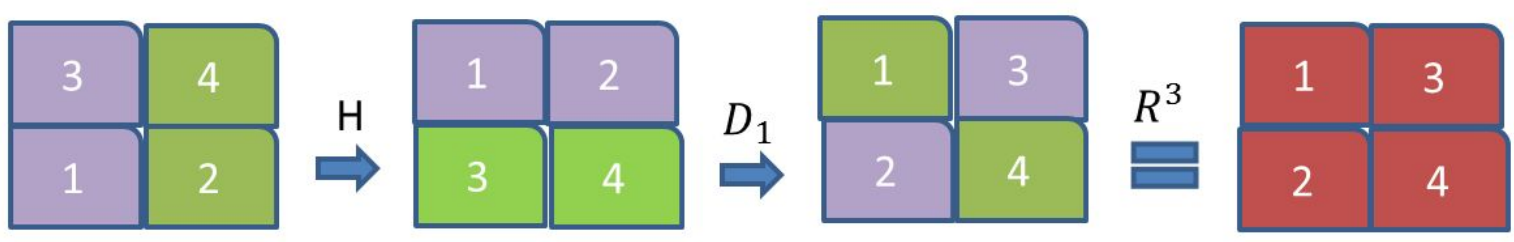

Figura 1.11: Movimiento $D_{1} H=R^{3}$

Inmediatamente otro grupo, mejoró la notación al percatarse de que la rotación de $360^{\circ}$, volvía a la posición inicial, es decir que $R_{4}=I$, de modo que resultaban 8 movimientos en lugar de 9. Otro grupo indicó que para simplicar notación, se podría usar $\left(R_{1}\right)^{2}$ en lugar de la rotación de $180^{\circ}$, y así con los demás. Este grupo usó la siguiente notación:

$D_{1}$ : que consiste en dejar 1 y 4 fijos e intercambiar 2 y 3 ,

$D_{2}$ : que consiste en dejar 2 y 3 fijos e intercambiar 1 y 4 .

$R$ : que corresponde a una rotación de $90^{\circ}$.

$R^{2}$ : que corresponde a una rotación de $180^{\circ}$.

$R^{3}$ : que corresponde a una rotación de $270^{\circ}$.

$H$ : que consiste en cambiar 1 con 3 y 2 con 4 .

$V$ : que consiste en cambiar 1 con 2 y 3 con 4 .

I: que consiste en dejar todo igual 
$\mathrm{Al}$ realizar la composición de los movimientos y formar la tabla del grupo, nuevamente al igual que en la consigna anterior, cada vez que querían ver el resultado, ponían la figura en la posición identidad. La docente intervino, impulsándolos a realizar las combinaciones, desde cualquier posición en que se hallara la figura, más fácilmente, usando la última posición. Los estudiantes dudaron un poco, pues no se sentían cómodos haciéndolo, sin embargo, comprobaron que los resultados se mantenían.

Otra observación a recalcar es que dos de los grupos de trabajo, concluyeron que no resultaba ser un grupo dado que al realizar la combinación $D_{1} \circ H$, vea figura 1.11 , se obtenía la diagonal doble de la consigna anterior $D$, la cual cambiaba a la vez: 1 con 2 y 3 con 4 , sin percatarse que esta correspondía al movimiento $R_{270}=R^{3}$, lo que fue aclarado por otro grupo.

Con miras a conocimientos a desarrollar luego, se les solicitó que verificaran que $D_{1} \circ R^{3}=R \circ D_{1}$, como puede verse en figuras 1.12 y 1.13, con la idea de conducirlos al caso general de un polígono regular de $n$ lados, a probar luego, que indica que si $S$ es la simetría que pasa por el vértice 1 , se tiene que

$$
S R^{k}=R^{-k} S, \quad \text { para todo } k=1, \cdots, n .
$$
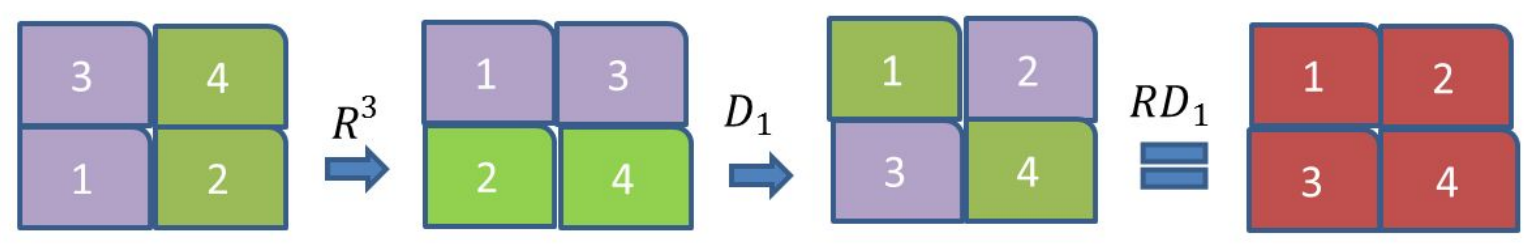

Figura 1.12: Movimiento $D_{1} \circ R^{3}$
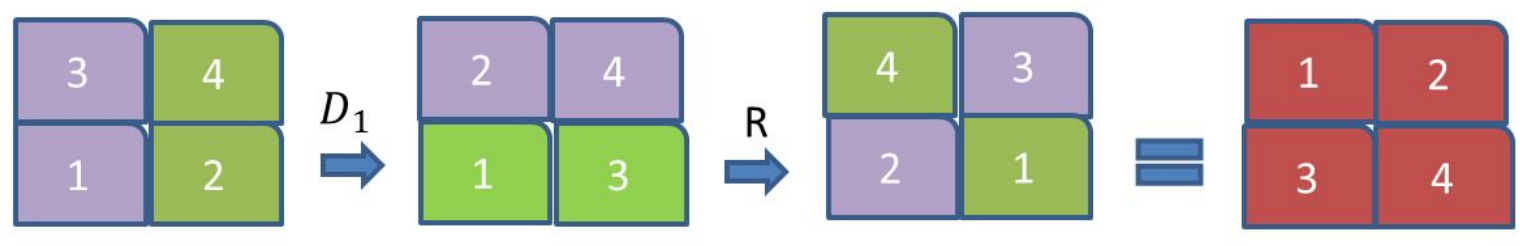

Figura 1.13: Movimiento $R \circ D_{1}$ 


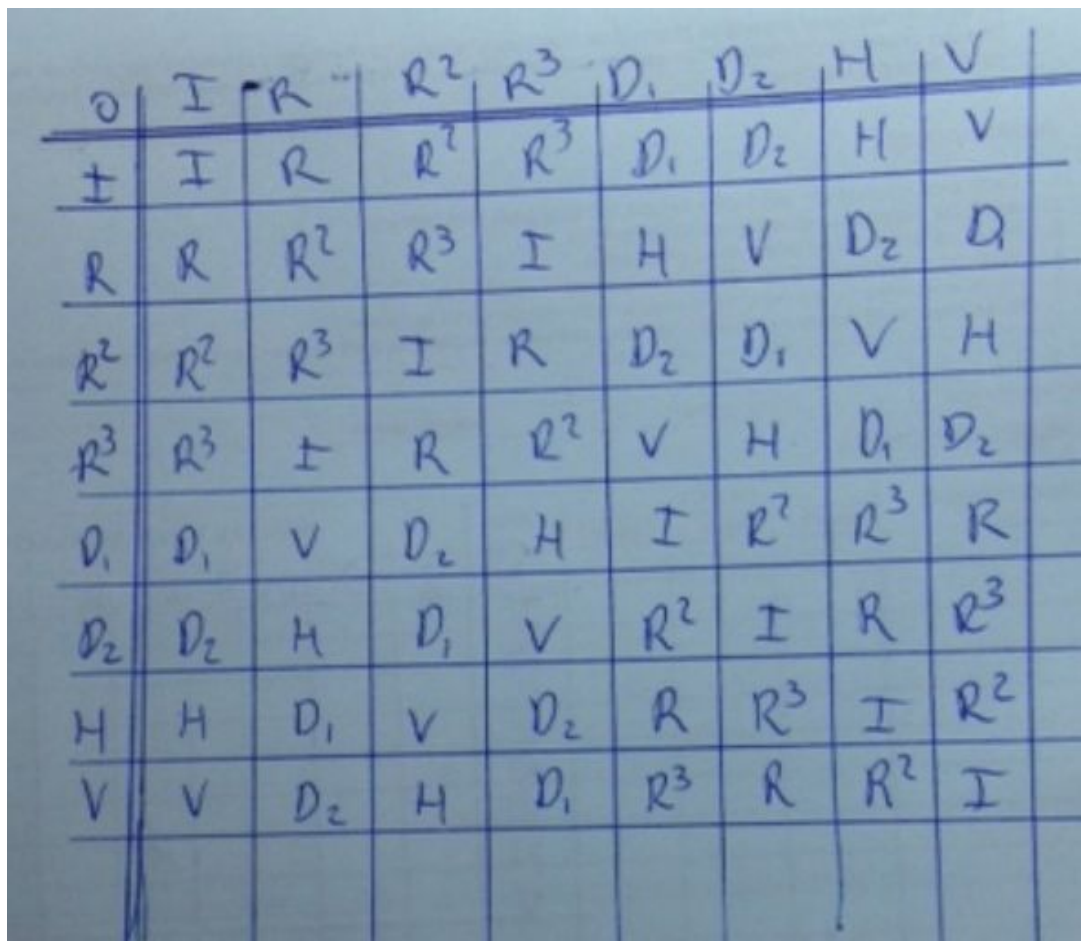

Figura 1.14: Tabla del grupo diedro $D_{2}$

Finalmente, todos lograron completar la tabla del grupo, y se les indicó que habían descubierto uno de los grupos más conocidos, el grupo diedro denotado por $D_{4}$, por los 4 vértices del polígono, el cual tiene 8 elementos. Uno de los grupos presentó la tabla dada en la figura 1.14.

En esta siguiente consigna, se les solicita hacer una variación al problema anterior, con miras a una generalización, con menos cubos, pero con movimientos similares: simetrías y rotaciones. Se trata de las simetrías del triángulo.

\section{Consigna 5: Menos cubos, movimientos similares}

Tome solamente tres cubos, numerados del 1 al 3, y posiciónelos como si fueran los vértices de un triángulo equilátero. Defina movimientos como simetrías con respecto a las alturas imaginarias y rotaciones, ¿de cuántos grados deberían ser las rotaciones?

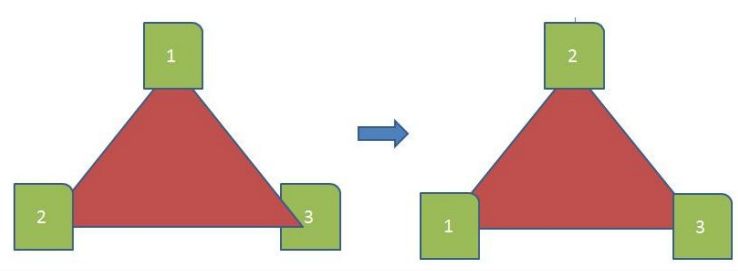

Construya una tabla de un grupo $(G, \circ)$ donde la operación $\circ$ es la composición de estos movimientos y $G$ es el conjunto formado por los todos los posibles movimientos. Identifique 
cuál movimiento funciona como identidad y cuáles son movimientos inversos. ¿Será conmutativa la operación? ¿De cuántos elementos resultó el grupo? ¿Y el anterior?

Los integrantes de los grupos, en forma diferentes, usaron la creatividad y versatilidad del material, para simular los movimientos del triángulo. Uno de ellos simuló en triángulo equilátero como muestra la figura 1.15 .

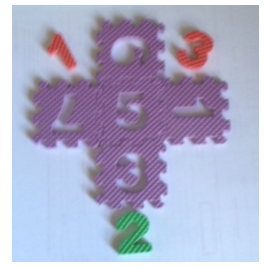

Figura 1.15: Representación de un triángulo

Así, la rotación de $90^{\circ}$ y la simetría con respecto a la altura sobre el vértice $1, A_{1}$, la representaron respectivamente, como se muestra en las figuras 1.16 y 1.17 .

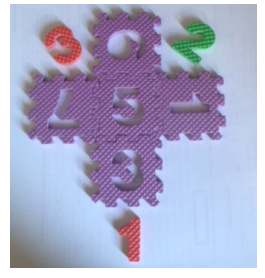

Figura 1.16: Rotación de $90^{\circ}$

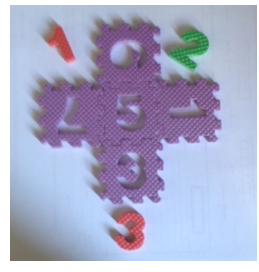

Figura 1.17: Simetría respecto a vértice 1

Sin embargo, cuando empezaron a hacer las composiciones, se les complicó la representación usada con el material, cuando los números no estaban en la posición inicial desde la identidad. Se dieron discusiones sobre si lo que importaba era el orden de los números que se movían de posición, o la posición de los vértices en los que los números se iban moviendo. Analizaron los dos casos, llegando a la conclusión de que lo que resultaba ser un grupo, era posicionar los vértices y a partir de ahí determinar las combinaciones de los movimientos. Por ejemplo, al realizar el movimiento $A_{2} R$, es decir después de una rotación de $90^{\circ}$, hacer una simetría con respecto al vértice 2 , tuvieron dificultad al decidir entre cuál de las dos figuras 1.18 ó 1.19, lo representaba.

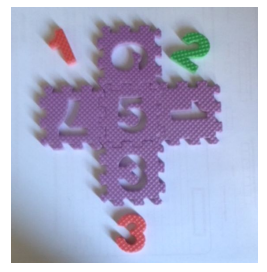

Figura 1.18: Representación errónea de $A_{2} R$

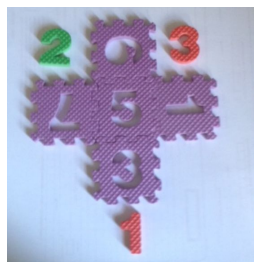

Figura 1.19: Representación correcta de $A_{2} R$

Es por esto, que resultó más adecuada la representación planteada por otro de los grupos, que usó el material, de modo que la posición quedara establecida desde el inicio, usándolo de la manera que 
muestra la figura 1.20, donde al sacar los números queda la forma del número en su posición inicial.

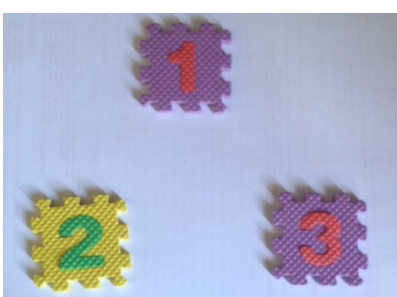

Figura 1.20: Rotación de $90^{\circ}$

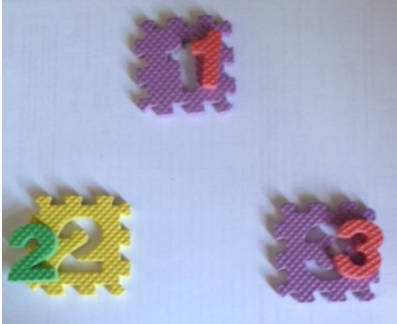

Figura 1.21: Simetría respecto a vértice 1

En este caso, quedan más claras las combinaciones de los movimientos, pues las posiciones de los vértices quedan plasmados con el "agujero" del número faltante. Así, el movimiento $A_{2} R$, se puede ver fácilmente.

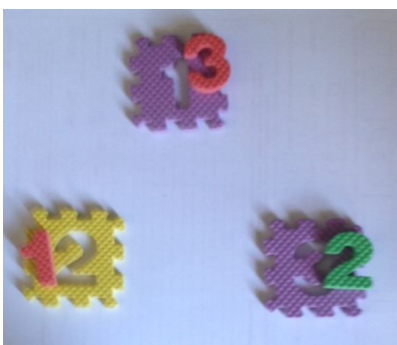

Figura 1.22: Rotación de $90^{\circ}$

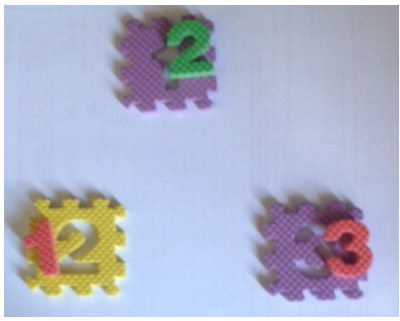

Figura 1.23: Representación de $A_{2} R$

Se adjunta en figura 1.24 la tabla creada por uno de los grupos.

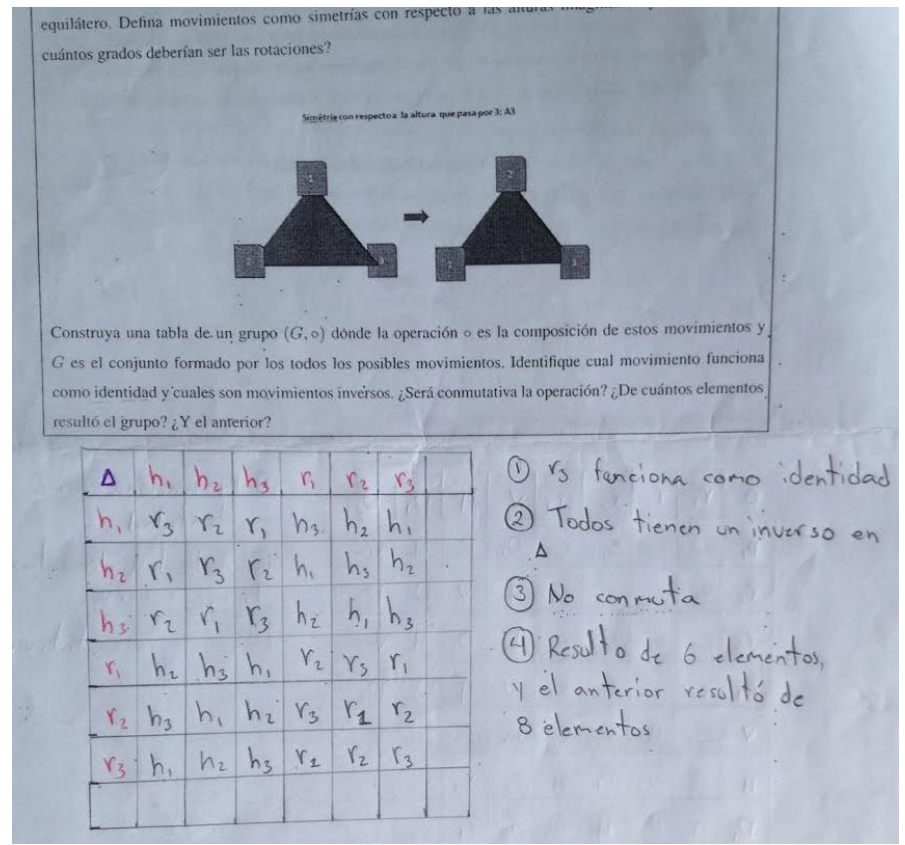


Figura 1.24: Tabla del grupo de simetrías del triángulo

La siguiente consigna, es para hacer en la casa. Esta involucra hacer una variación al problema anterior, con un número mayor de cubos, con un grado de dificultad mayor, para así así lograr una generalización. Es decir, la intención es que construya un grupo con movimientos similares, pero ahora en un heptágono.

\section{Consigna 6: Hacia una generalización}

Ahora suponga que tiene 7 cubos numerados del 1 al 7. Defina movimientos básicos de modo que al combinarlos, obtenga un grupo. ¿De cuántos elementos estará constituido este grupo, si incluye simetrías y rotaciones?

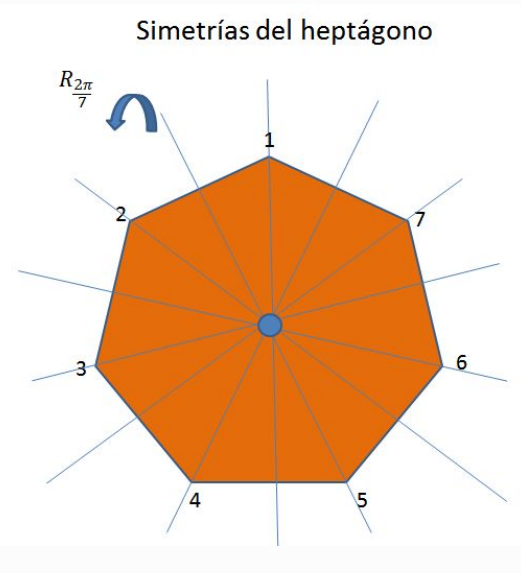

Para este último, se le deja al estudiante que defina las simetrías y rotaciones, también es una tarea para la casa. Finalmente se espera que construya la tabla. La idea es que la siguiente clase se haga la demostración formal de los resultados y pueda aplicarlos a estas dos consignas.

Ya para esta altura, los estudiantes rápidamente concluyeron que este resultaría en un grupo de orden 14, y que realizar todos los movimientos y sus combinaciones, iba a resultar tedioso. De ahí la necesidad, surgida de ellos mismos, de buscar formas alternativas de deducir la tabla de cualquier grupo en que se incluyeran rotaciones y simetrías a un polígono regular de $\mathrm{n}$ lados, buscando algún software dinámico.

\subsubsection{Consignas del bloque 3:}

En este bloque, la docente instituciona las ideas y construcciones de tablas del bloque anterior, probando que para cualquier polígono regular de $n$ lados, se puede formar un grupo, si se toma como operación la composición de rotaciones y simetrías. Pero antes de iniciar la prueba del teorema, se les presentó la siguiente definición, para ir introduciéndolos en el lenguaje formal de la prueba. 


\section{Definición 1.1}

Dado un conjunto $A$ del plano, denotaremos por $S(A)$ al conjunto de todos los movimientos que se le pueden aplicar a $A$, dejándolo invariante, es decir que no cambian su forma. Más específicamente,

$$
S(A)=\{M: \text { M es un movimiento y } M(A)=A\}
$$

Tipos de estos movimientos, pueden ser traslaciones, rotaciones, simetrías respecto a una recta, etc, como las aplicadas en las consignas anteriores. A este conjunto de movimientos $S(A)$, se le llama "simetrías del plano".

\section{Consigna 7: Prueba formal del grupo de las simetrías del plano}

El siguiente teorema da una prueba formal, que indica que al aplicar movimientos como los realizados en las consignas del bloque anterior, al cuadrado, hexágono y demás polígonos regulares, se obtiene un grupo con la operación composición. Fórmese en los grupos de trabajo, y siga paso a paso la demostración dada por la docente.

\section{Teorema 1.1}

El conjunto de las simetrías de un conjunto $A$ del plano, es un grupo, con la operación composición de movimientos.

Prueba: Sea $A$ un conjunto del plano y sea $S(A)=\{M:$ M es un movimiento y $M(A)=A\}$. Sean $M, N \in S(A)$. Entonces $M(A)=A$ y $N(A)=A$. Luego

$$
(M \circ N)(A)=M(N(A))=M(A)=A,
$$

por lo que $M \circ N \in S(A)$, es decir se cumple la cerradura. La asociatividad es válida, pues si $M, N, L \in$ $S(A)$, entonces $((M \circ N) \circ L)(A)=A=(M \circ(N \circ L))(A)$. El neutro es el movimiento de no hacer nada, es decir no mover a $A$, que denotaremos con $I$, de identidad y el inverso es el movimiento de "devolverse". El inverso de $M$ es un elemento de $S(A)$, pues

$$
M^{-1}(A)=M^{-1}(M(A))=\left(M^{-1} \circ M\right)(A)=I(A)=A
$$

Veamos uno de los ejemplos realizados en la actividad 1, antes de probar el teorema que formaliza los resultados hallados. Para mayor comodidad en la notación, de ahora en adelante obviaremos el símbolo de la composición $\circ$, de modo que, para referirse a $M \circ N$, simplemente usaremos la notación MN. 
Consigna 8: Formalizando el grupo de las simetrías del triángulo

En la consigna 5, ustedes realizaron los movimientos de simetrías y rotaciones para un triángulo. Vuelva a reconstruir los movimientos hechos, y compárelos con la demostración formal dada por la docente.

\section{Ejemplo 1.1 (Simetrías del triángulo)}

Considere un triángulo equilátero con vértices en las posiciones 1,2 y 3.

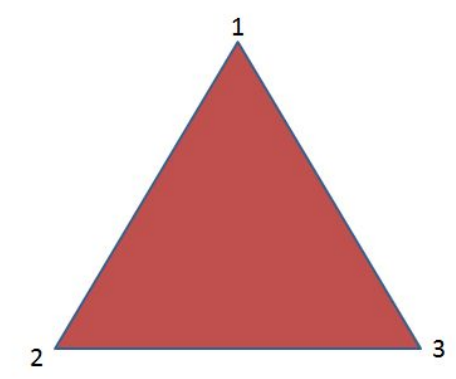

Sea $R$ la rotación de $120^{\circ}$. Observe que $R^{2}$ es la rotación de $240^{\circ}$, mientras que $R^{3}$ es la rotación de $360^{\circ}$, que denotaremos con $I$, de identidad. Vea figura 1.25 .

Sea ahora $S_{1}$ la simetría con respecto a la altura que pasa por el vértice $1, S_{2}$ la simetría con respecto a la altura que pasa por el vértice 2 y $S_{3}$ la simetría con respecto a la altura que pasa por el vértice 3. Observe que $R \circ S_{1}=S_{3}$, mientras que $R^{2} \circ S_{1}=S_{2}$.

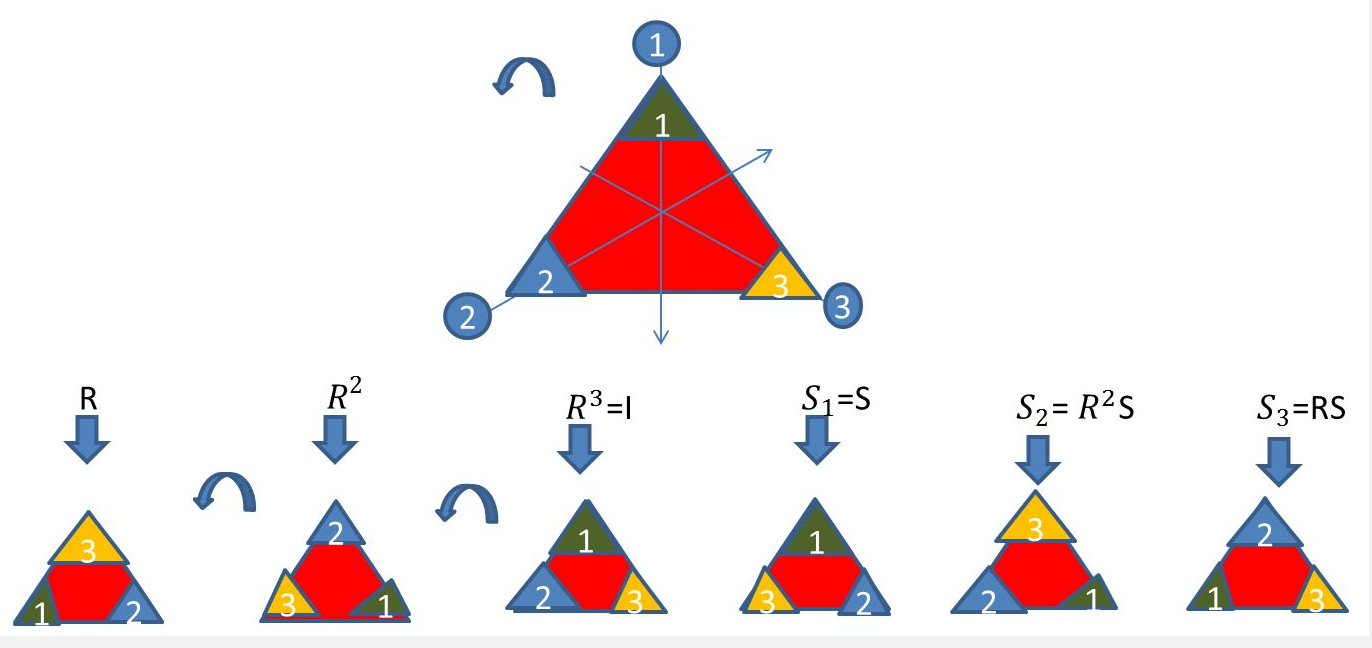

Figura 1.25: Simetrías del triángulo

De modo que $S_{2}$ y $S_{3}$ se pueden expresar como composiciones de las rotaciones y de la simetría con respecto a la altura que pasa por el vértice 1. Para simplificar la notación, llamaremos a esta última, simplemente $S$ y obviaremos el símbolo de la operación composición, es decir en lugar de escribir $R \circ S_{1}$, escribiremos $R S$, como se mencionó anteriormente. 
La tabla para este grupo, está dada en la figura 1.26.

\begin{tabular}{l|l|c|c|c|c|c}
\multicolumn{1}{c|}{0} & $\mathrm{I}$ & $\mathrm{R}$ & $\mathrm{R}^{2}$ & $\mathrm{~S}$ & $\mathrm{RS}$ & $\mathrm{R}^{2} \mathrm{~S}$ \\
\hline $\mathrm{I}$ & $\mathrm{I}$ & $\mathrm{R}$ & $\mathrm{R}^{2}$ & $\mathrm{~S}$ & $\mathrm{RS}$ & $\mathrm{R}^{2} \mathrm{~S}$ \\
\hline $\mathrm{R}$ & $\mathrm{R}$ & $\mathrm{R}^{2}$ & $\mathrm{I}$ & $\mathrm{RS}$ & $\mathrm{R}^{2} \mathrm{~S}$ & $\mathrm{~S}$ \\
\hline $\mathrm{R}^{2}$ & $\mathrm{R}^{2}$ & $\mathrm{I}$ & $\mathrm{R}$ & $\mathrm{R}^{2} \mathrm{~S}$ & $\mathrm{~S}$ & $\mathrm{RS}$ \\
\hline $\mathrm{S}$ & $\mathrm{S}$ & $\mathrm{R}^{2} \mathrm{~S}$ & $\mathrm{RS}$ & $\mathrm{I}$ & $\mathrm{R}^{2}$ & $\mathrm{R}$ \\
\hline $\mathrm{RS}$ & $\mathrm{RS}$ & $\mathrm{S}$ & $\mathrm{R}^{2} \mathrm{~S}$ & $\mathrm{R}$ & $\mathrm{I}$ & $\mathrm{R}^{2}$ \\
\hline $\mathrm{R}^{2} \mathrm{~S}$ & $\mathrm{R}^{2} \mathrm{~S}$ & $\mathrm{RS}$ & $\mathrm{S}$ & $\mathrm{R}^{2}$ & $\mathrm{R}$ & $\mathrm{I}$ \\
\hline
\end{tabular}

Figura 1.26: Tabla del grupo de simetrías del triángulo

De este modo, las simetrías del triángulo tiene 6 elementos y está dado por el conjunto:

$$
S(A)=\left\{I, R, R^{2}, S, R S, R^{2} S\right\}
$$

Ahora, se generaliza formalmente el grupo de simetrías de cualquier polígono regular en el siguiente teorema.

\section{Teorema 1.2}

Sea $P(n)$ un polígono regular con $n$ vértices. Sea $R$ la rotación de $\frac{2 \pi}{n}$ radianes alrededor del centro del polígono y sea $S$ la simetría con respecto a una recta que pasa por el centro de $P(n)$ y uno de sus vértices. Entonces el conjunto de simetrías $S(P(n))$, es un grupo con la operación composición de movimientos, que consta de $2 n$ elementos. A este grupo se le llama grupo diedro del polígono y se acostumbra a denotarlo con $D_{2 n}$.

$$
D_{2 n}=\left\{I, R, R^{2}, \cdots, R^{n-1}, S, R S, R^{2} S, \cdots, R^{n-1} S\right\}
$$

Prueba: Las ideas de esta prueba, fueron tomadas de Dorronsoro(1996). Es claro que $R^{2}$ es una rotación de $\frac{4 \pi}{n}, R^{3}$ es una rotación de $\frac{6 \pi}{n}$, y continuando así, obtenemos que $R^{n}$ es una rotación de $\frac{2 n \pi}{n}$, que lleva al polígono a la posición inicial. De modo que si $j$ denota el vértice de la posición $j$, al plicarle una rotación de $\frac{4 \pi}{n}$ una vez, este vértice pasa a la posición $j+1$, es decir se tiene que

$$
R(j)=j+1, \quad \text { para } \quad j=1, \cdots, n-1 \quad \text { y } \quad R(n)=1
$$

Veamos ahora qué hacen las simetrías a cualquiera de los vértices. Sin pérdida de generalidad, podemos suponer que $S$ es la simetría con respecto a la recta que pasa por el vértice 1. 
Como puede verse en la figura 1.27 , esta simetría cambia el vértice 2 con el vértice $n$, el 3 con $n-1$, el 4 con $n-2$, y en general

$$
S(j)=n-(j-2), \quad \text { para } \quad j=2, \cdots, n, \quad \mathrm{y} \quad S(1)=1
$$

Mostraremos que $R S, R^{2} S, \cdots, R^{n-1} S$, son todas las simetrías con respecto a las rectas que pasan por lo vértices del polígono.

Sea $L$ la recta que pasa por uno de los vértices y por el punto medio del lado que une los vértices 1 y 2 , es decir la recta que está girada $\frac{\pi}{n}$ con respecto a la recta $S$.

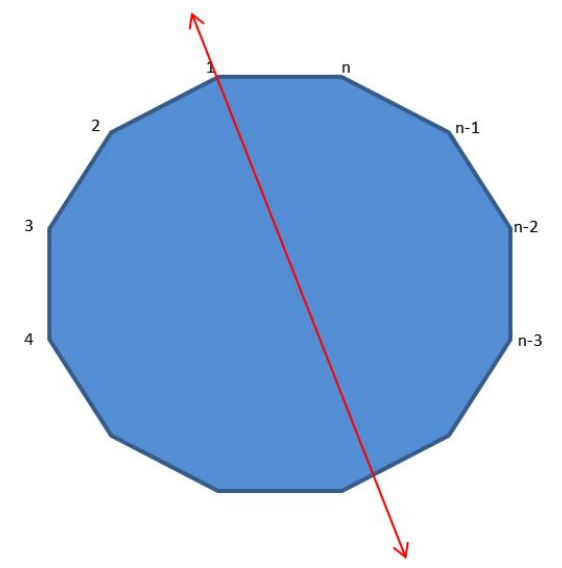

Figura 1.27: Simetría con respecto a la recta que pasa por vértice 1

Es claro, según figura 1.28, que $L$ cambia los vértices 1 con 2,3 con $n, 4$ con $n-1$, y en general sigue la siguiente regla:

$$
L(j)=n-(j-3), \quad \text { para } \quad j=3, \cdots, n \quad \mathrm{y} \quad L(1)=2, \quad L(2)=1
$$

Mostraremos que la simetría con respecto a $L$ corresponde al movimiento $R S$. En efecto

$$
R S(j)=R(S(j))=R(n-j+2)=n-j+3, \quad \text { para } \quad j=3, \cdots, n
$$

pues $R(j)=j+1$ para $j=1, \cdots, n-1$. Además

$$
R S(1)=R(1)=2, \quad R S(2)=R(n)=1, \quad y \quad R S(n)=R(1)=3 ，
$$

de modo qe efectivamente la simetría con respecto a $L$, es el mismo movimiento dado por la composición $R S$.

Consideramos ahora la simetría con respecto a la recta $S_{2}$ que pasa por el vértice 2. Como puede verse en la figura 1.29, ésta simetría cambia los vértices de acuerdo con la siguiente regla:

$$
S_{2}(j)=n-(j-4), \quad \text { para } \quad j=4, \cdots, n \quad y \quad S_{2}(1)=3, \quad S_{2}(2)=2, \quad S_{2}(3)=1
$$

Pero esto, nuevamente, coincide con $R^{2} S$, pues

$$
R^{2} S(j)=R^{2}(S(j))=R(n-j+2)=n-j+4=S_{2}(j), \quad \text { para } \quad j=1, \cdots, n
$$


De modo que tenemos que $R S=L$ y que $R^{2} S=S_{2}$. Generalizando por un proceso inductivo, si denotamos por $S_{k}$ a la simetría con respecto a la recta que pasa por el vértice $k$, tenemos que

$$
R^{k} S=S_{k} \quad \text { para } \quad k=2, \cdots, n-1
$$

de modo que entonces obtenemos que $R S, R^{2} S, \cdots, R^{n-1} S$, son todas las simetrías con respecto a las rectas que pasan por los vértices del polígono. Con esto el conjunto tiene todas las rotaciones y simetrías del polígono, es decir está completo, no le falta ninguna rotación ni ninguna simetría.

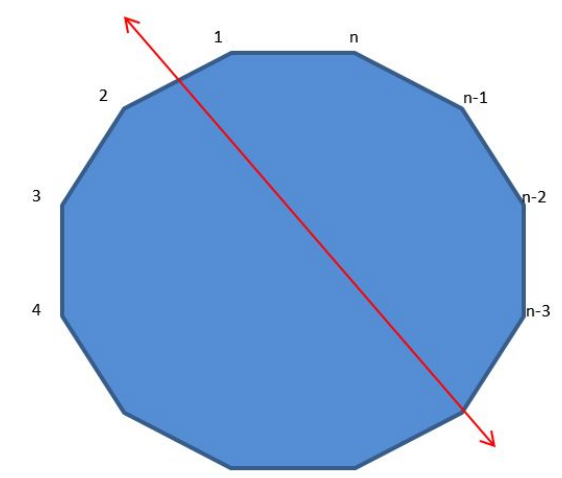

Figura 1.28: Simetría con respecto a la recta entre los vértices 1 y 2

Ahora solo falta probar que $D_{2 n}$ es efectivamente un grupo. El neutro es claramente $I=R^{n}$, pues esta vuelve a poner en la posición original al polígono, después de cualquier rotación dado que $R^{n}(j)=$ $j, \forall n=1, \cdots, n$, es decir que

$$
R^{n} R^{k}=R^{k}, \quad \forall k=1, \cdots, n .
$$

Pero también este funciona como identidad para cualquier movimiento que involucre una simetría, pues

$$
R^{n} R^{k} S=R^{k} S \quad \forall k=1, \cdots, n .
$$

La asociatividad se hereda de la operación composición, que es válida en este caso.

Por otro lado, para analizar la existencia de los inversos, podemos ver que

$$
R^{k}(j)=j+k, \quad \text { y que } \quad R^{-k}(j)=j-k, \quad \forall k=1, \cdots, n
$$

por ser la rotación en sentido contrario. De modo que el inverso de cualquier rotación $R^{k}$ es $R^{-k}$, pues

$$
\left(R^{k} R^{-k}\right)(j)=R^{k}(j-k)=j, \quad \forall k=1, \cdots, n
$$

Por otro lado, probaremos, como la intuición indica después de las actividades realizadas, que el inverso de cualquier simetría es ella misma, pues mueve los vértices y los devuelve a su posición. Para ello mostraremos antes, una propiedad muy útil que ocurre con la composición de estos movimientos, y es que

$$
S R^{k}=R^{-k} S, \quad \forall k=1, \cdots, n .
$$

En efecto,

$$
\left(S R^{k}\right)(j)=S\left(R^{k}(j)\right)=S(j+k)=n-(j+k-2), \quad \text { para } \quad j=1, \cdots, n, \quad \forall k=1, \cdots, n
$$

mientras que

$$
\left(R^{-k} S\right)(j)=R^{-k}(S(j))=R^{-k}(n-(j-2))=R^{-k}(n-j+2)=n-j+2-k,
$$


para $j=1, \cdots, n, \quad \forall k=1, \cdots, n$, por lo que ambas coinciden. Esto nos indica que el inverso de $R^{k} S$ es el mismo $R^{k} S$. En efecto

$$
\left(R^{k} S\right)\left(R^{k} S\right)=R^{k}\left(S R^{k}\right) S=R^{k}\left(R^{-k} S\right) S=\left(R^{k} R^{-k}\right) S^{2}=I
$$

La propiedad es muy útil para completar la tabla de $D_{2 n}$, y así finalmente verificar la cerradura. Se concluye que este es un grupo.

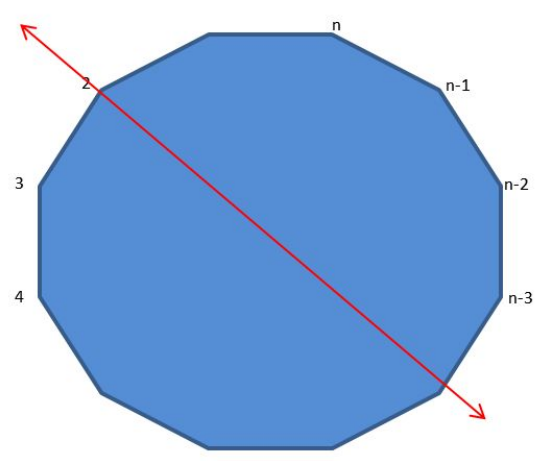

Figura 1.29: Simetría con respecto a la recta que pasa por el vértice 2

Después de realizar la prueba, se les dejó de tarea de construir la tabla, usando la propiedad

$$
R^{k} S=S_{k} \quad \text { para } \quad k=2, \cdots, n-1,
$$

en un polígono de mayor cantidad de vértices. También se les pidió simular los movimientos usando algún software dinámico.

\section{Consigna 9: Un polígono con mayor número de vértices}

Ahora suponga que tiene 9 cubos numerados del 1 al 9. Defina movimientos básicos de modo que al combinarlos, obtenga un grupo. ¿De cuántos elementos estará constituido este grupo, si incluye simetrías y rotaciones? 


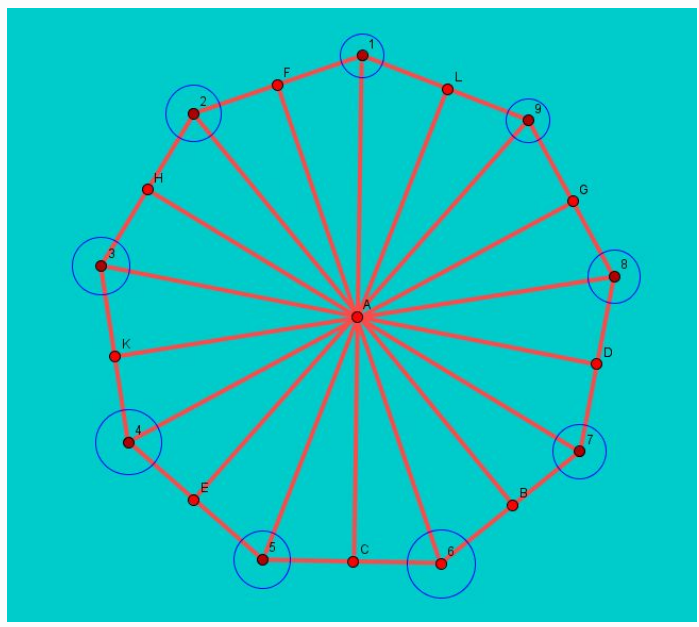

Uno de los grupos presentó su solución, un grupo de orden 18, como puede verse en la figura 1.30 .

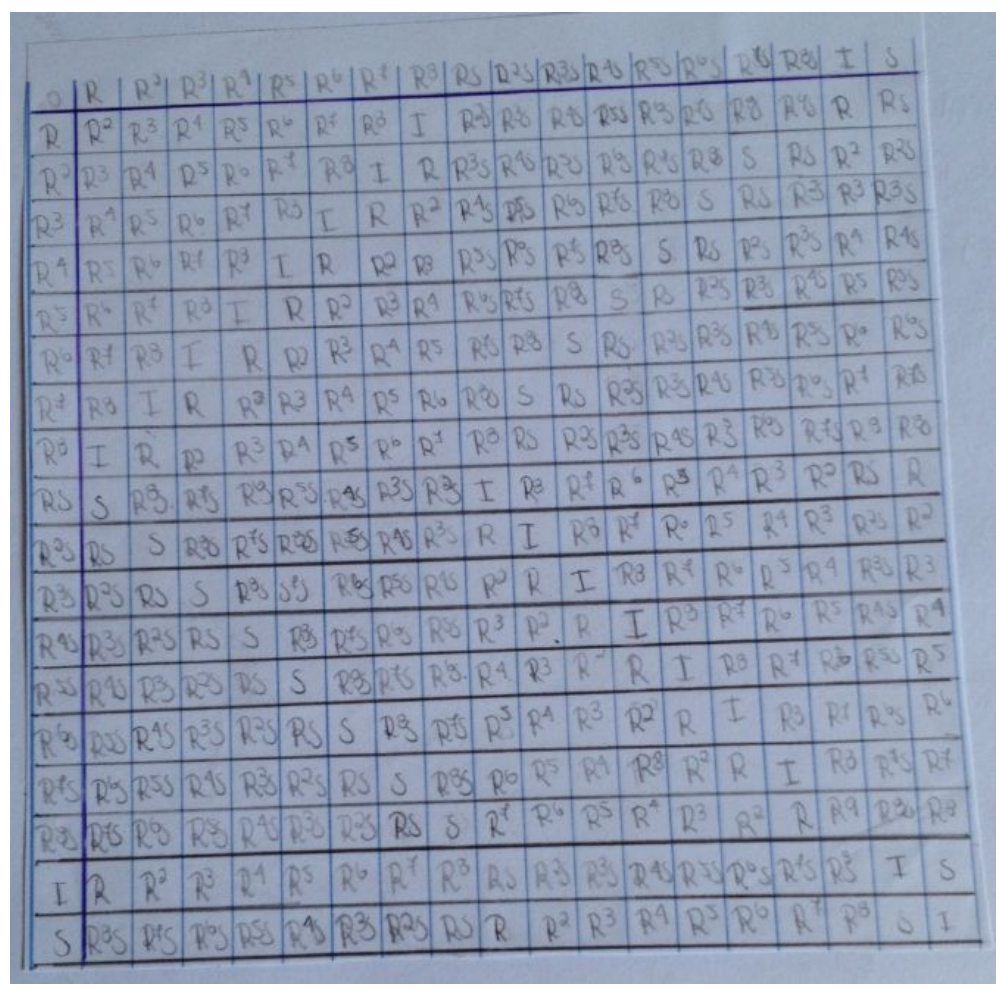

Figura 1.30: Grupo de simetrías del nonágono

\subsubsection{Consignas del bloque 4: Actividades de reforzamiento extra-clase}


- ¿Cuál podría haber sido la intención de la docente al plantearles estas consignas?

Consideramos que la docente buscó que comprendiéramos el concepto de grupo de una manera más dinámica y que nos permitiera profundizar más en el tema. Además como nuestra formación es en Enseñanza de la Matemática la docente nos está brindando una idea distinta para abordar la introducción a un tema, como lo son la consignas, al trabajar nosotros mismos con ellas no permitió analizar sobre su utilidad y los temas en que se podría aplicar.

A continuación se le dejaron tres consignas para realizar fuera de clase. En esta última parte de la actividad, se espera que los estudiantes hagan una valoración de toda la experiencia, ahora en calidad de futuros docentes, de manera que reflexionen sobre la misma, su funcionalidad y mejoras, respondiendo a las preguntas de la siguiente consigna. Se les motivó para que contestaran con toda la transparencia y sinceridad posible, dieran críticas constructivas, a nivel de profesores sugeriendo mejoras a otra profesora.

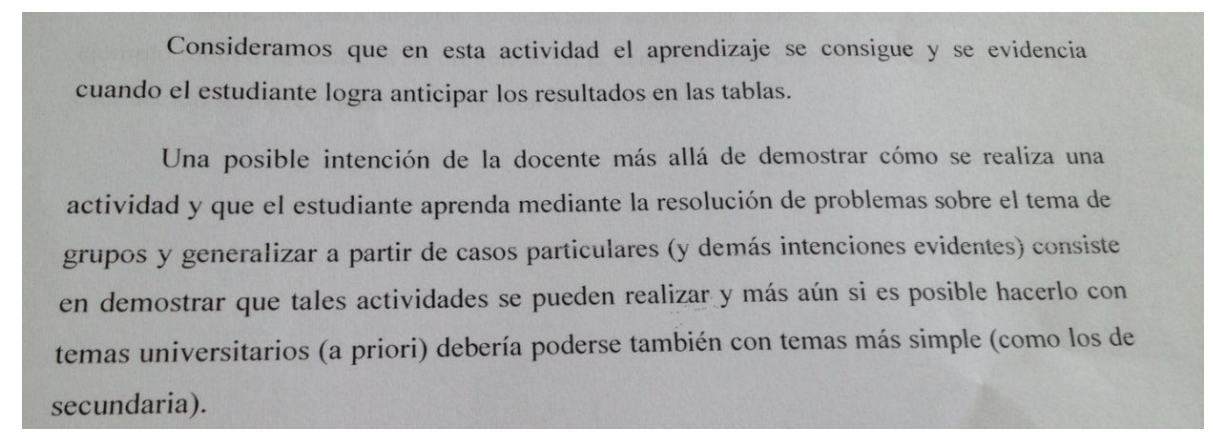

Figura 1.31: Comentarios sobre la intención de la actividad

\section{Consigna 10:}

Conteste las preguntas: ¿Se logra aprender con las actividades propuestas? ¿He aprendido? ¿Cuál podría haber sido la intención de la docente al plantearnos estas consignas? ¿Porqué no dar simplemente la definición y luego todos esos ejemplos? ¿No debería la docente desarrollar cada uno de estos ejercicios en lugar de ponernos a nosotros a hacer tal cantidad de trabajo? ¿Se podría mejorar la gestión de la clase? ¿Se usaron los recursos adecuados? ¿El tiempo estuvo bien gestionado?

\footnotetext{
- ¿Se usaron los recursos adecuados?

Consideramos que el material que usamos es idóneo pues nos permitió usarlo como modelo para simular las relaciones que ocurren entre los elementos de los conjuntos que forman un grupo, además el material permitió que la actividad se realizara a modo de juego.
}

Figura 1.32: Comentarios sobre el uso de material concreto 
- ¿Cuál podría haber sido la intención de la docente al plantearles estas consignas?

Consideramos que la docente buscó que comprendiéramos el concepto de grupo de una manera más dinámica y que nos permitiera profundizar más en el tema. Además como nuestra formación es en Enseñanza de la Matemática la docente nos está brindando una idea distinta para abordar la introducción a un tema, como lo son la consignas, al trabajar nosotros mismos con ellas no permitió analizar sobre su utilidad y los temas en que se podría aplicar.

Figura 1.33: Comentarios sobre el uso de material concreto

Esta consigna la respondieron fuera de la clase, de modo que tuvieran plena tranquilidad de dar sus respuestas. Algunas de las respuestas se dieron a continuación. Con respecto a la intención de las consignas y de la actividad en sí, se agregan algunos comentarios, en las figuras 1.33 y 1.31.

El material además de económico resulta adecuado y motivante. Una cualidad

importante del material es su facilidad de uso (cualidad que no necesariamente poseen las

TICs, que en muchos casos requieren de instrucciones más elaboradas).

Figura 1.34: Comentarios sobre el uso de material concreto

Con respecto al uso de material concreto, en general fue muy bien aceptado, les resultó una clase diferente y amena, como lo expresaron. Unos de los estudiantes comentó: " gracias, la clase estuvo muy linda". Algunos de los estudiantes que en clases tradicionales, se caracterizan por sentarse atrás muestran poca participación e interés, en esta actividad estuvieron participativos por el entusiasmo mostrado. Cabe mencionar que se destacaron en los logros y alcances de la actividad, inclusive terminando primero que otros grupos. Algunos comentarios al respecto son los de las figuras $1.32 \mathrm{y}$ 1.34 .

Con respecto a si la actividad logró su objetivo académico, es decir si logró el aprendizaje del concepto de grupo, también se lograron resultados y comentarios muy positivos, como puede verse en figuras 1.35 y 1.36 .

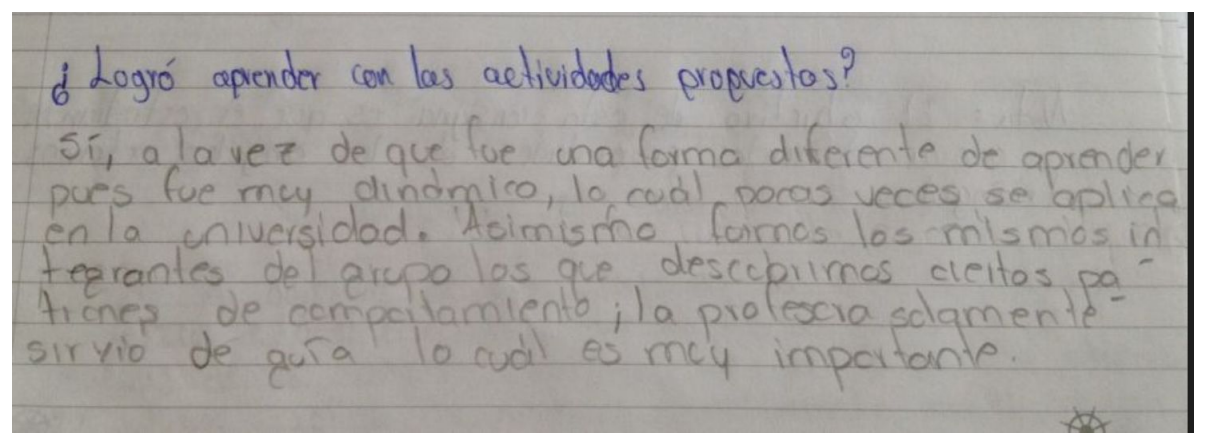

Figura 1.35: Comentario sobre el aprendizaje logrado 
- ¿Logró aprender con las actividades propuestas?

En grupo comentamos lo útiles que fueron las consignas en la construcción y comprensión del concepto de grupo, creemos que interrogantes como las planteadas en la consigna 2 son muy útiles para uno como estudiante ya que pueden aplicarse a cualquier tema para tener seguridad de que se ha interiorizado realmente. Los distintos ejemplos permitieron identificar patrones e ideas necesarias para demostrar las características y propiedades de los grupos.

Figura 1.36: Comentario sobre el aprendizaje logrado

Finalmente, con respecto a mejoras, hubo un consenso en el hecho de que el tiempo estuvo limitado. otro grupo comentó que faltó más comentarios a nivel de todo el grupo, y sobre como resolvieron cada uno las consignas.

\section{- ¿Se podría mejorar la gestión de la clase?}

Creemos que sería importante que se pudieran compartir los resultados obtenidos en los distintos grupos con el fin de observar distintas caminos para resolver un mismo ejercicio y enriquecerse con el trabajo de otros.

Figura 1.37: Sugerencias de mejora a la actividad

La siguiente consigna solicita que los estudiantes lean e investiguen sobre el tema de creación de problemas, se les da un artículo reciente para su lectura, y se les solicita investigar sobre dos más relacionados al tema, que ellos deben buscar en la web, e indicar su dirección y el porqué de su escogencia.

7. Con sus propias palabras defina qué es una función.

8. ¿Hay diferencia entre una relación y una función? ¿Cual?

- Consigna 2

Considere dos conjuntos, $M=\{$ mama $\}$ y $H=\{$ hijo1, hijoz, hijo3, hijo4 $\}$. Realice un

diagrama de Venn donde se relacionen $M \rightarrow B$ y otro $H \rightarrow M$ y responda lo siguiente

1. ¿Representan una funcion?

2. Explique con sus palabras porque son o no funciones.

Figura 1.38: Tareas creadas por los estudiantes para el tema de funciones 
- Consigna 3

Construya una función con elementos de la vida cotidiana siguiendo las siguientes pautas:

1. Asigne un nombre a la función.

2. Determine dominio, codominio y nómbrelos.

3. Construya el criterio que relacione elementos del dominio con elementos del codominio.

- Consigna 4

Utilice los dados facilitados para formar relaciones, uno de los dados será quien arroja los valores del dominio y el otro los valores del codominio.

Lance simultaneamente ambos dados 10 veces, cada lanzamiento representa la relación de dos elementos. Ahora

1. Dibuje un diagrama de Venn con los resultados obtenidos.

2. ¿Obtuvo una función? ¿Por quê??

- Consigna 5

Con los dados realice tres diagramas de Venn de igual forma que en la consigna anterior. intercámbielos con algún compañero para que responda si representan o no funciones.

Figura 1.39: Continuación...

\section{Consigna 11:}

Lea el artículo relacionado a creación y plantamiento de problemas de Malaspina, U. (2013). "Nuevos horizontes matemáticos mediante variaciones de un problema". Unión, 35, 135-143. Haga un resumen del artículo y un comentario. Luego busque dos artículos más relacionados al tema y coméntelos.

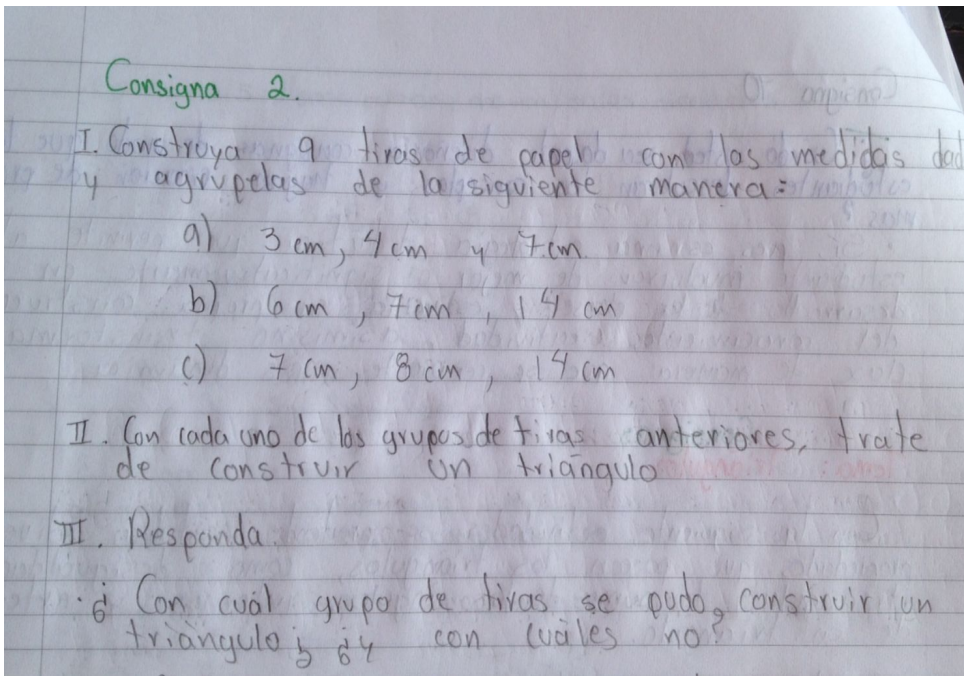

Figura 1.40: Tareas creadas por los estudiantes para introducir triángulos rectángulos 
La siguiente consigna está dirigida a que ellos implementen algunas tareas sobre algún tema de secundaria, con ayuda de las lecturas seleccionadas y la práctica llevada a cabo en las consignas de la actividad realizada.

\section{Consigna 12:}

Cuando usted sea docente, ¿diseñaría consignas de modo que los estudiantes descubran los conceptos y hagan creación de problemas? Diseñe 5 consignas para enseñar algún tema de secundaria.

\section{Ejemplo: Ubicar números irracionales en la recta numérica (ya se conoce el} conjunto numérico).

1. La aparición del concepto formal de "irracional" tarda aproximadamente 1800 años en la historia matemática, esto porque no existia un consenso general sobre su definición como conjunto numérico. En este caso, uno de los primeros personajes en enfrentar tal discusión fue Pitágoras y su regla general. ¿Qué propone el teorema de Pitágoras? Obtenga tres ejemplos que cumplan el teorema con números enteros $(\mathrm{a}, \mathrm{b}, \mathrm{c})$. ¿Qué sucede con el caso de $\mathrm{a}=1$ y $\mathrm{b}=1$ ?

2. La recta numérica se utiliza para ubicar todos los números reales. En ese sentido, cualquier persona deberia tener la capacidad de colocar ejemplos de ese conjunto en la recta sin utilizar aproximaciones con calculadora (Ante este ejercicio utilice únicamente regla y compás). Para iniciar ubique únicamente números enteros y racionales $(3 / 2,-4,2 / 5,3)$.

3. Ante la discusión de aproximación de los números irracionales, haga un listado de 5 elementos de ese conjunto cuya ubicación exacta se desconoce pero que tienen la facilidad de ser ubicar entre 2 números enteros. ¿Cuál método de aproximación utilizaria?

4. Dibuje la recta numérica con una recta vertical en la posición del cero $(x=0)$. Tome uno de los casos de la consigna \#1 y ubiquelo como par ordenado $(x, y)$. Luego trace el segmento que se forma entre el par $(x, y)$ y $(0,0)$. Utilice el compás para dibujar un círculo de radio " $r$ " (cuya medida es igual a la distancia entre el par ordenado

Figura 1.41: Tareas creadas por los estudiantes para ubicar números en la recta real

Esta fue una de las consignas más productivas desde el punto de vista de su futuro profesional como docentes. Los estudiantes plantearon varios consignas para diferentes problemas. Sin embargo, es claro que aún falta mayor madurez para el diseño de tareas con un objetivo concreto, pero esto es algo que requiere tiempo y dedicación. Algunas de las tareas creadas se adjuntan en las figuras 1.38, 1.40 y 1.41. 
En la figura 1.38, los estudiantes escogieron el tema de funciones; ciertamente un tema que es difícil de enseñar y el cual encierra conceptos muy delicados y sutiles, y que además es la base del análisis y cursos universitarios, por lo que en secundaria, su enseñanza y aprendizaje es fundamental. Interesante notar como este grupo propone el uso de dados para que se realicen lanzamientos de ellos y analizar si se obtiene una función. Por otro lado proponen también buscar funciones en la vida real, contextualizando la matemática.

En la figura 1.40, también usan material concreto, en este caso tiras de papel para que los estudiantes intenten formar triángulos rectángulos. Faltaría sin embargo, una consigna que los lleve a una generalización sobre la relación entre los lados del triángulo, que los acerque al teorema de Pitágoras.

En la figura 1.41 se trata de realizar ubicaciones de números reales en la recta numérica. Primero construyen una consigna que los haga pensar como ubicar un número irracional que está entre dos enteros, pero no se les guía a como hacerlo. Luego en la consigna siguiente, se les pide usar regla y compás y se les da el método tradicional de hacerlo.

\subsection{Consideraciones Finales}

Se presentó una secuencia de tareas diseñadas con un doble propósito: lograr una asimilación sólida del concepto de grupo como base para las otras estructuras algebraicas: anillo y campo. Sin embargo, la actividad no llegó hasta aquí, sino que además se logró cierto nivel de reflexión sobre la actividad realizada, desde la prespectiva de estudiantes que pronto, serán docentes. Se deben realizar más actividades de este tipo, de modo que estas reflexiones mejoren cada día. Con respecto al primer objetivo, se puede decir que se logró ampliamente; los estudiantes asimilaron muy bien la existencia del neutro, su unicidad y su función dentro de un grupo. Así mismo, comprendieron la idea de lo que son los inversos así como de su unicidad, y de que cada elemento tiene su inverso. Esto se evidenció en los resultados de una prueba escrita que se les aplicó una semana después de concluidas las actividades. La mayoría de los estudiantes, lograron resultados muy por encima, de otros semestres donde se les impartió el mismo concepto, pero con una clase magistral. También, quedó clara la idea de isomorfismo entre grupos, concepto que en otras ocasiones, había tenido fuertes limitaciones y mostrado carencias de conexiones.

Con respecto al objetivo dos, que era lograr la reflexión sobre la matemática, podría concluir que aún falta mucho en este aspecto, sin embargo es claro que esto lleva tiempo y madurez, requiere de más experiencias similares y de una constante que debe mantenerse en el aula. Se requiere además, despertar en los estudiantes, la necesidad de búsqueda de información y realización de lecturas que no solo respondan a una tarea que se debe cumplir. Se debe insistir en estos aspectos formadores.

Es importante señalar que el diseño de un conjunto de tareas debe tener un propósito claro, y que el docente debe realizar un planeamiento de ellas, tratando de predecir el desarrollo que puede darse en la práctica, pero que debe tenerse muy claro, que en la realidad de aula, pueden presentarse situaciones que no resulten como se ha planeado. El profesor debe estar anuente a tomar medidas para encauzar a los estudiantes en el logro del objetivo planteado.

Por último, es importante recalcar la responsabilidad de todos los docentes que de alguna manera participamos en la formación inicial de profesores de matemática para secundaria, de incluir actividades que desarrollen la competencia de reflexión sobre la actividad matemática y que una forma de lograrlo, es modelando con actividades prácticas en la mismo aula y en la enseñanza de los propios cursos de matemática. 


\section{Bibliografía}

[1] Castillo, S. (2008) Propuesta pedagógica basada en el constructivismo para el uso óptimo de las TIC en la enseñanza y aprendizaje de la matemática. Revista Latinoamericana de Investigación en Matemática Educativa (2008) 11(2): 171-194

[2] Dorronsoro, J. (1996). Números, grupos y anillos. Addison-Wesley Ibereoamericana, S.A.

[3] Ellerton, N. F. (2013). Engaging pre-service middle-school teacher-education students in mathematical problem posing: development of an active learning framework.. Educational Studies in Mathematics, 83(1), 87-101.

[4] Font, V. (2011a). Competencias profesionales en la formación inicial de profesores de matemáticas de secundaria. Unión - Revista Iberoamericana de Educación Matemática, 26, 9-25.

[5] Giménez, J., Font, V. y Vanegas, Y. (2013). Designing Professional Tasks for Didactical Analysis as a research process, en C. Margolinas (Ed.), Task Design in Mathematics Education. Proceedings of ICMI Study 22, (Vol. 1, 581-590). Oxford: ICMI

[6] Kilpatrick, J.; Gómez, P. y Rico, L. (1995). Educación matemática. México: Grupo Editorial Iberoamérica.

[7] Malaspina, U. (2013). Nuevos horizontes matemáticos mediante variaciones de un problema. Unión, $35,135-143$.

[8] Margolinas, C (2013), Task Design in Mathematics Education. Proceedings of ICMI Study 22, (Vol. 1, 581-590). Oxford: ICMI

[9] Mason, J. y Johnston-Wilder, S. (2004). Designing and Using Mathematical Tasks. London: Tarquin.

[10] Rico, L.(2004 ) Reflexiones sobre la formación inicial del profesor de matemáticas de secundaria. Profesorado, revista de currículum y formación del profesorado, 8 (1).

[11] Rubio, N. (2012). Competencia del profesorado en el análisis didáctico de prácticas, objetos y procesos matemático. Tesis doctoral no publicada, Universitat de Barcelona, España.

[12] Silverman, J. y Thompson, P. (2008). Toward a framework for the development of mathematical knowledge for teaching. Journal of mathematics teacher education, 11(6), 499-511.

[13] Singer. F. M. y Voica, C. (2013). A problem-solving conceptual framework and its implications in designing problem-posing tasks. Educational Studies in Mathematics, 83(1), 9-26.

[14] Van Harpen, X. Y, y Presmeg, N. C. (2013). An investigation of relationships between students mathematical problem-posing abilities and their mathematical content knowledge. Educational Studies in Mathematics, 83(1), 117-132. 